\begin{tabular}{|l|c|c|c|c|}
\hline $\begin{array}{l}\text { Cuadernos de Investigación Geográfica } \\
\text { Geographical Research Letters }\end{array}$ & 2019 & N $^{\circ} 45(1)$ & pp. 87-121 & eISSN 1697-9540 \\
\hline
\end{tabular}

\title{
VARIACIÓN DE LOS DOMINIOS FORESTAL Y HERBÁCEO EN EL PAISAJE VEGETAL DE LA PENÍNSULA IBÉRICA EN LOS ÚLTIMOS 20.000 AÑOS. IMPORTANCIA DEL EFECTO DE LOS GRANDES HERBÍVOROS SOBRE LA VEGETACIÓN
}

\author{
G. MONTSERRAT-MARTÍ1*, D. GÓMEZ-GARCÍA ${ }^{2}$ \\ ${ }^{1}$ Instituto Pirenaico de Ecología, Consejo Superior de Investigaciones Científicas (IPE-CSIC), \\ Campus de Aula Dei, Apartado 13.034, 50080 Zaragoza, España. \\ ${ }^{2}$ Instituto Pirenaico de Ecología, Consejo Superior de Investigaciones Científicas (IPE-CSIC), \\ Avda. Nuestra Señora de la Victoria, 16, 22700 Jaca (Huesca), España.
}

\begin{abstract}
RESUMEN. Los mega-herbívoros (masa corporal superior a $1000 \mathrm{~kg}$ ) contribuyen a estructurar el paisaje vegetal por medio del consumo, la rotura y el desarraigo de numerosas plantas leñosas, tendiendo a transformar las zonas boscosas en mosaicos de pasto, matorral y bosque. Estos mosaicos ayudan a mantener una elevada biodiversidad ya que facilitan la persistencia de plantas y animales de ambientes esteparios desarbolados y forestales densos que alternaron en los paisajes europeos siguiendo los ciclos glaciales del Pleistoceno. La desaparición de los mega-herbívoros de la península Ibérica a final del Pleistoceno seguramente provocó una cascada de efectos ecológicos que todavía desconocemos. También ignoramos las causas de esta desaparición, si fueron naturales o antrópicas, por lo que tampoco podemos saber si los paisajes vegetales que se desarrollaron posteriormente, en el Holoceno, son de origen natural o antrópico.
\end{abstract}

El objetivo de esta revisión es explorar en la literatura científica la hipótesis de que la transformación del paisaje por el hombre tras su llegada a Europa comenzaría por la caza intensiva de los grandes herbívoros, causando su desaparición o la disminución drástica de sus poblaciones. La consiguiente reducción del efecto de estos animales sobre las plantas leñosas, que había sido muy intenso en los anteriores interglaciares, propiciaría una marcada recuperación forestal al final del Pleistoceno y comienzos del Holoceno. Los bosques densos se extenderían mermando los pastos y herbívoros de espacios abiertos, que fueron el principal sustento de Homo sapiens en el Pleistoceno. Esta limitación obligaría a los humanos a cambiar su dieta para sobrevivir y, en un periodo de tiempo relativamente corto, a adoptar un nuevo modo de vida basado en la ganadería y la agricultura. 
Los resultados de la revisión no permiten avalar ni rechazar la hipótesis planteada, ni siquiera alguna de las cuestiones más relevantes derivadas de ella. Varios fundamentos de esta hipótesis se debaten actualmente y están dando lugar a interpretaciones contrapuestas.

Se discuten los aspectos ecológicos más relevantes encontrados, se proponen algunas ideas para su interpretación y se enfatizan las limitaciones de la información paleo-ecológica publicada para responder a preguntas tan generales como las que planteamos.

\section{Variation of the forest and herbaceous domains in the landscape of the Iberian Peninsula in the last 20,000 years. Importance of the effect of large herbivores on vegetation}

ABSTRACT. Mega-herbivores (body mass greater than $1000 \mathrm{~kg}$ ) help structuring the plant landscape through consumption, breakage and uprooting of numerous woody plants, tending to transform wooded areas into grass, shrub and forest mosaics. These mosaics contribute to maintain a high biodiversity, since they enable the persistence of plants and animals of dense forests and treeless steppes, which alternated in the European landscapes following the glacial cycles of the Pleistocene. The disappearance of the mega-herbivores from the Iberian Peninsula at the end of the Pleistocene probably caused a cascade of ecological effects that remains unknown. We also do not know if the causes of their disappearance were natural or anthropogenic, and consequently it is not know if the plant landscapes that developed later, in the Holocene, have a natural or anthropogenic origin.

The aim of this review is to explore in the scientific literature the hypothesis that the transformation of the natural landscape by humans began after their arrival in Europe through the intensive hunting of large herbivores, until their disappearance or extreme reduction. Consequently, the forest recovery of the end of the Pleistocene and beginning of the Holocene might have taken place without the containment that these animals would have exerted on woody vegetation in the previous interglacials. As a result, dense forests would spread over large territories reducing pastures and herbivores of open spaces, which were the sustenance of Homo sapiens in the Pleistocene. This limitation would force humans to change their diet to survive and, in a relatively short period of time, to adopt a new way of life-style based on livestock and agriculture.

The results of the review do not allow us to support or reject the hypothesis raised, or even any of the most relevant issues arising from it. Some fundamental grounds of this hypothesis are currently debated, and are giving rise to contrasting interpretations.

The most relevant ecological aspects found after the literature review are discussed, some ideas are proposed for their interpretation, and the limitations 
of the paleo-ecological information available in the literature to answer general questions such as those posed here, are emphasized.

Palabras clave: Transformación del paisaje vegetal, consumo por grandes herbívoros, final del Pleistoceno, Holoceno, península Ibérica.

Key words: Landscape transformation, consumption by large herbivores, late Pleistocene, Holocene, Iberian Peninsula.

*Correpondencia: Gabriel Montserrat-Martí, Instituto Pirenaico de Ecología, Consejo Superior de Investigaciones Científicas (IPE-CSIC), Campus de Aula Dei, Apartado 13.034, 50080 Zaragoza, España. E-mail: gmmarti@ipe.csic.es

\section{Introducción}

El paisaje vegetal ha experimentado grandes transformaciones en la península Ibérica durante los últimos 20 milenios (González-Sampériz et al., 2010, 2017; Carrión et al., 2010 a). Entre los mayores cambios hay que destacar el de los dominios forestales respecto a las formaciones de pasto-matorral. Hace 20.000 años en Europa dominaban las estepas sin árboles (Binney et al., 2017) y en el Holoceno dominaron los bosques hasta que en el Neolítico se produjo una fuerte transformación del paisaje con la llegada de la agricultura, la expansión de los pastos para el ganado doméstico y los asentamientos urbanos (Kaal et al., 2011; Innes et al., 2013). Esta transformación ha continuado hasta la actualidad, acabando en la práctica con los paisajes vírgenes en Europa (Schnitzler, 2014).

La reconstrucción de la vegetación y del paisaje vegetal en los periodos anteriores al Neolítico se suele apoyar en las características del clima, del suelo y, en mucha menor medida, en otros factores como son los regímenes de perturbación (Bond y Keeley, 2005; Gill, 2014). De estos últimos, el fuego ostenta la máxima consideración (Bond et al., 2005). Otros factores de perturbación, causados por los herbívoros de tamaños grande y mediano, se han menoscabado (Barnosky et al., 2016), aunque algunos autores les conceden gran importancia (Zimov et al., 1995; Coope, 2000; Rule et al., 2012; Gill et al., 2012, 2013; Gill 2014, entre otros).

En la descripción del paisaje, desde la perspectiva del uso por los vertebrados herbívoros, interesa distinguir la ocupación espacial de las zonas forestales (bosques y matorrales) de las dominadas por plantas herbáceas, además de las zonas de transición o ecotonos (bosques poco densos, pastos con árboles dispersos, etc.). Esa distinción es relevante porque los herbívoros que se alimentan en pastos despejados o poco arbolados (pastadores) han sido muy importantes en la península Ibérica durante los últimos millones de años (Agustí y Antón, 2002) y en la actualidad constituyen la mayoría de los herbívoros domésticos (García-González, 2008). El dominio de uno u otro tipo de vegetación en el paisaje vegetal no sólo depende del clima, sino también de los regímenes de perturbación y su intensi- 
dad, las propiedades del suelo, etc. Además, con el aumento del estrés (por limitaciones al crecimiento vegetal derivadas del clima, los nutrientes, el agua, la luz, etc., Grime, 1979) se requiere un régimen de perturbaciones menos intenso para favorecer el dominio de las comunidades de plantas herbáceas y leñosas de pequeña talla sobre las comunidades de árboles y arbustos (Fig. 1). Es decir, se favorecen los pastos y los herbívoros pastadores en contra de los bosques-matorrales y los herbívoros ramoneadores (Hofmann, 1989). Un buen ejemplo de esta relación es el descenso del límite del bosque que se asocia al aumento de la perturbación antrópica en las altas montañas (González-Guerrero et al., 2017).

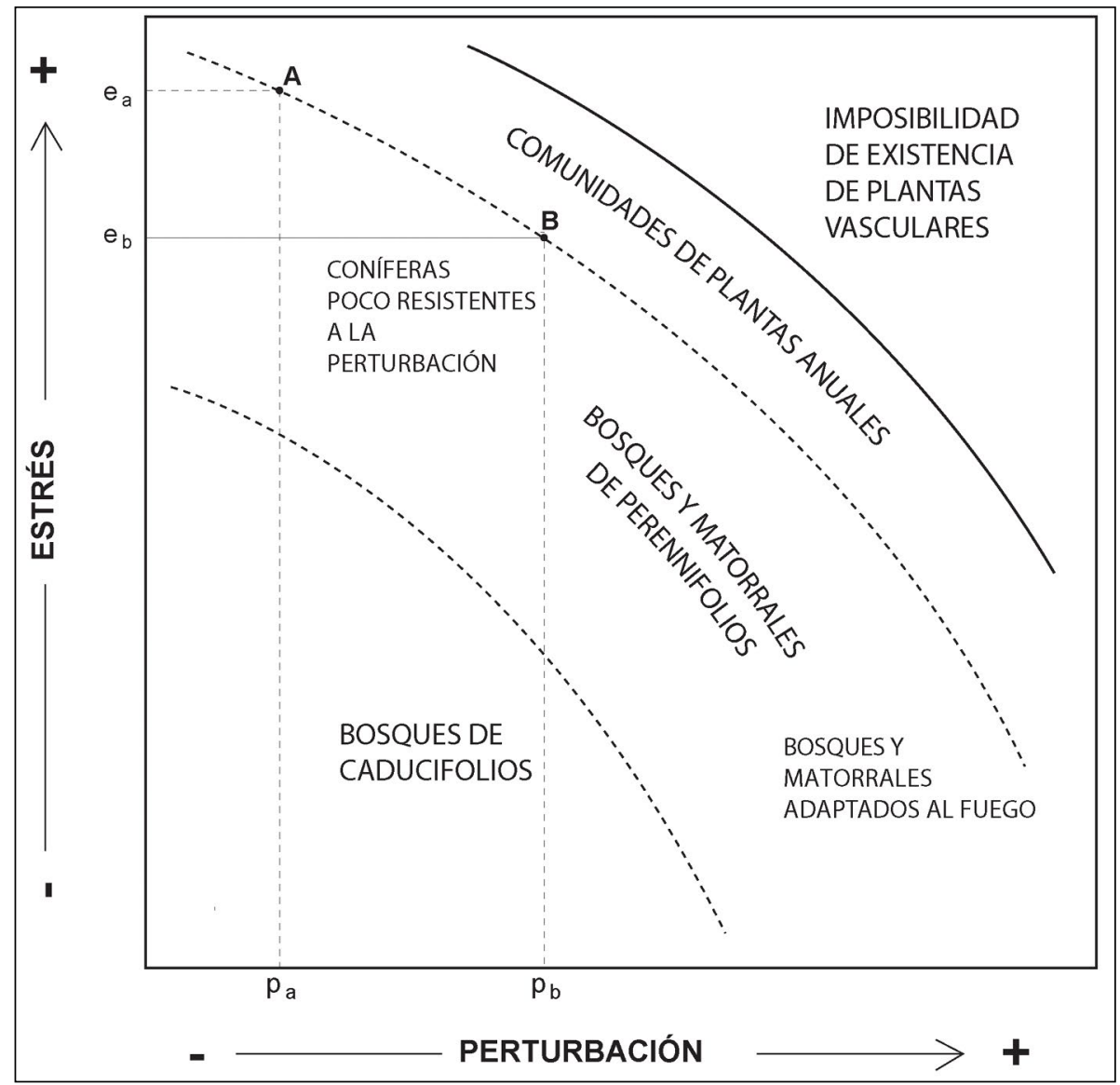

Figura 1. Esquema de los dominios generales de la vegetación natural de la península Ibérica según los regímenes de estrés y perturbación que soportan durante el año. Los puntos $A$ y $B$ representan dos situaciones de límite altitudinal de los árboles sometidas a distintos regímenes de perturbación a mayor y menor altitud, respectivamente. Se han considerado los conceptos de estrés y perturbación de Grime (1979).

El objetivo de este trabajo es explorar en la literatura científica la hipótesis de que la transformación del paisaje natural por nuestra especie comenzó poco después de su 
llegada a Europa, hace unos 40-45.000 años (Hervella et al., 2016). El desencadenante de esa transformación habría sido la caza intensiva de los grandes herbívoros gracias a las técnicas sofisticadas de caza que Homo sapiens había comenzado a desarrollar antes de su expansión por Europa y que perfeccionó sustancialmente en los siguientes milenios (Flodin, 1999; Shea, 2006; Churchill y Rhodes, 2009). La disminución o incluso desaparición de las manadas de herbívoros de tamaño grande y medio determinaría que, a final del Pleistoceno y comienzo del Holoceno, la recuperación de árboles y arbustos se produjera sin la contención que habrían ejercido estos animales en los interglaciares anteriores (ver Bakker et al., 2016 para un planteamiento de hipótesis similar). Los bosques densos se extenderían por grandes territorios en detrimento de los pastos, limitando aún más las poblaciones de herbívoros pastadores de espacios abiertos, que habían sido el principal sustento de Homo sapiens y H. neanderthalensis en tiempos anteriores. Esta limitación obligaría a los humanos a cambiar su dieta para sobrevivir y, en un periodo de tiempo relativamente corto, a adoptar un nuevo modo de vida basado en la ganadería y la agricultura. Es importante resolver las preguntas derivadas de esta hipótesis, entre otras muchas cosas, para poder saber si los paisajes vegetales que se produjeron durante el Holoceno en Europa, desprovistos de mega-herbívoros (masa corporal de los adultos de más de $1000 \mathrm{Kg}$, Owen-Smith, 1987), son naturales o de origen antrópico (Birks 2005) y, en consecuencia, qué tipo de medidas se han de aplicar para la conservación y restauración de los distintos paisajes de la península Ibérica que consideramos más naturales. Las ideas que aquí se discuten no son nuevas. Algunos autores, como Owen-Smith (1987), Zimov et al. (1995, 2012), Johnson (2009), Doughty et al. (2010), Rule et al. (2012), Doughty y Malhi (2013), Gill (2014), Barnosky et al. (2016), Johnson et al. (2016), Bakker et al. (2016), etc., han propuesto ideas similares. La originalidad del presente estudio se limita a situar el planteamiento general de la hipótesis en la península Ibérica en los últimos 20.000 años, así como discutir las implicaciones más importantes de dicha hipótesis para la interpretación, gestión y conservación de nuestros paisajes naturales.

\section{Revisión de la literatura}

\subsection{Paisaje vegetal del final del Pleistoceno y extinción de los grandes herbívoros}

El último pleniglaciar (24.000-14.600 años BP), que incluyó alguno de los episodios más fríos del Pleistoceno (Mix et al., 2001; Jouzel et al., 2007), es un buen punto de referencia para entender la relación que existía entre la vegetación, un clima extremo (alto estrés por frío y sequedad) y el efecto de los herbívoros de mediano y gran tamaño sobre la vegetación. Existe la idea clásica de que durante el último máximo glacial (LGM, en torno a 20.000 años BP) las estepas y tundras sin árboles cubrirían gran parte de la Europa no cubierta por el hielo, incluyendo el norte de la península Ibérica (Binney et al., 2017). El resto de la península estaría cubierto por estepas poco arboladas, bosques ralos de coníferas y numerosos refugios de vegetación mediterránea y de clima templado junto a la costa y en los valles interiores más abrigados (González-Sampériz et al., 2010). Se cree que durante el LGM no existían bosques extensos en Europa sino pequeñas poblaciones de árboles localizadas en enclaves favorables (Willis y van Andel, 2004). En los últimos años se ha cuestionado esta idea de inmensas estepas y tundras sin árboles 
que ocupaban gran parte de Europa y se ha generado un activo debate sobre la existencia de refugios de árboles por encima de los límites previamente considerados $\left(45^{\circ} \mathrm{N}\right.$ para los árboles de clima templado y de $46^{\circ} \mathrm{N}$ para todos los árboles en el $\mathrm{W}$ de Europa), es decir, de los refugios del sur de Europa (Willis y van Andel, 2004; Svenning et al., 2008; Tzedakis et al., 2013; Robin et al., 2016; Giesecke, 2016). Los partidarios de los refugios de especies arbóreas en el centro y norte de Europa se apoyan en evidencias paleo-ecológicas (Willis y van Andel, 2004; Juricková et al., 2014; Dering et al., 2016), en las predicciones de algunos modelos de nichos ecológicos (Svenning et al., 2008) y en estudios genéticos (Parducci et al., 2012; Dering et al., 2016). Las principales causas que se han propuesto para explicar la ausencia o escasez de árboles en los paisajes del LGM son la dureza del clima (frío y aridez), algunos fenómenos periglaciares, el efecto de los fuertes vientos, el mal drenaje de los suelos, las bajas concentraciones de $\mathrm{CO}_{2}$ en la atmósfera y el fuego de origen natural o causado por los humanos (Kolstrup 1990; Bredenkamp et al., 2002; Svenning et al., 2008; Kaplan et al., 2016). Se suele asumir que el efecto de los incendios sobre la flora leñosa en los periodos glaciales fue menos importante que en los interglaciares (Lawson et al., 2013; Power et al., 2008). Sin embargo, Kaplan et al. (2016) consideran que los fuegos de origen antrópico debieron ser fundamentales durante el LGM, dada la gran destreza de los humanos paleolíticos en el manejo del fuego que, en este caso, dirigirían a facilitar la caza, la recolección y la movilidad.

Es sorprendente que en la mayoría de las reconstrucciones de los paisajes vegetales del LGM se margine el efecto de los grandes herbívoros. Por ejemplo, Kaplan et al. (2016) le atribuyen un escaso efecto al valorar que la densidad de estos animales debía ser muy baja en los ambientes infértiles del LGM.

La extinción de gran parte de la megafauna entre el final del Pleistoceno y el principio del Holoceno ha despertado mucho interés entre los científicos durante más de un siglo (Smith et al., 2016; Ricánková et al., 2018). Este interés se reactivó cuando Martin (1967) planteó la caza como la causa más probable de la extinción, generando un activo debate que aún continúa (Koch y Barnosky, 2006; Smith et al., 2016; Ricánková et al., 2018). Algunos científicos sostienen que antes de su extinción muchas especies de la megafauna experimentaron un declive continuado que se ha correlacionado con importantes cambios climáticos (Guthrie, 2001; Nogués-Bravo et al., 2010; Carotenuto et al., 2016; Ricánková et al., 2018), otros, proponen que una intensa caza o la alteración de los hábitats por los humanos condujeron a la extinción de estos animales (Martin, 1967; Martin y Steadman, 1999; Surovell et al., 2005, 2016; Gill et al., 2009; Zimov et al., 2012, Sandom et al., 2014a) y, finalmente, el grupo más numeroso de autores suponen que la extinción se produjo por una combinación de ambas causas (Stuart, 2005, 2015; Koch y Barnosky 2006; Pushkina y Raia, 2008; MacDonald et al., 2012; Markova et al., 2013; Bartlett et al., 2016). Por otra parte, se ha descrito cómo la pérdida de la megafauna determinó una cascada de efectos ecológicos que afectaron profundamente a la estructura de la vegetación y la función de los ecosistemas (Johnson, 2009; Gill, 2014; Bakker et al., 2016; Mahli et al., 2016; Smith et al., 2016), las interacciones planta-animal (Guimaraes et al., 2008), los regímenes de fuego (Rule et al., 2012), los ciclos biogeoquímicos (Doughty y Malhi, 2013) y el clima (Doughty et al., 2010; Smith et al., 2010). 
Se considera que la extinción de la megafauna en Europa se produjo en dos fases: la primera comenzó con el declive poblacional de algunas especies a final del anterior interglaciar (Riss-Würm, 140.000-115.000 años BP) y concluyó con su extinción al inicio del último episodio glacial. Junto a otros animales más pequeños, se extinguieron los hipopótamos (Hippopotamus amphibius), los elefantes de colmillos rectos (Palaeoloxodon antiquus) y los rinocerontes esteparios (Stephanorhinus spp.) (Stuart, 2015). Todos ellos estaban poco adaptados al intenso frío de los episodios glaciales más severos por lo que sus últimos refugios europeos parece que se situaron en el sur del continente. La desaparición de sus últimas poblaciones parece que sucedió tras la llegada de Homo sapiens a Europa, aproximadamente hace unos 45.000 años (Owen-Smith, 1987; Koch y Barnosky, 2006; Stuart, 2015). La segunda fase comenzó con el declive de algunas especies tras el último máximo glacial y concluyó con su extinción en momentos distintos, entre el final del Pleistoceno y el Holoceno (Fig. 2). En ella se extinguieron, junto a otras especies de menor talla, el rinoceronte lanudo (Coelodonta antiquitatis) y el mamut (Mammuthus primigenius), ambos muy adaptados a las extremas condiciones climáticas glaciales por lo que mantuvieron sus últimos refugios en Eurasia central y norte de Siberia (Markova et al., 2013).

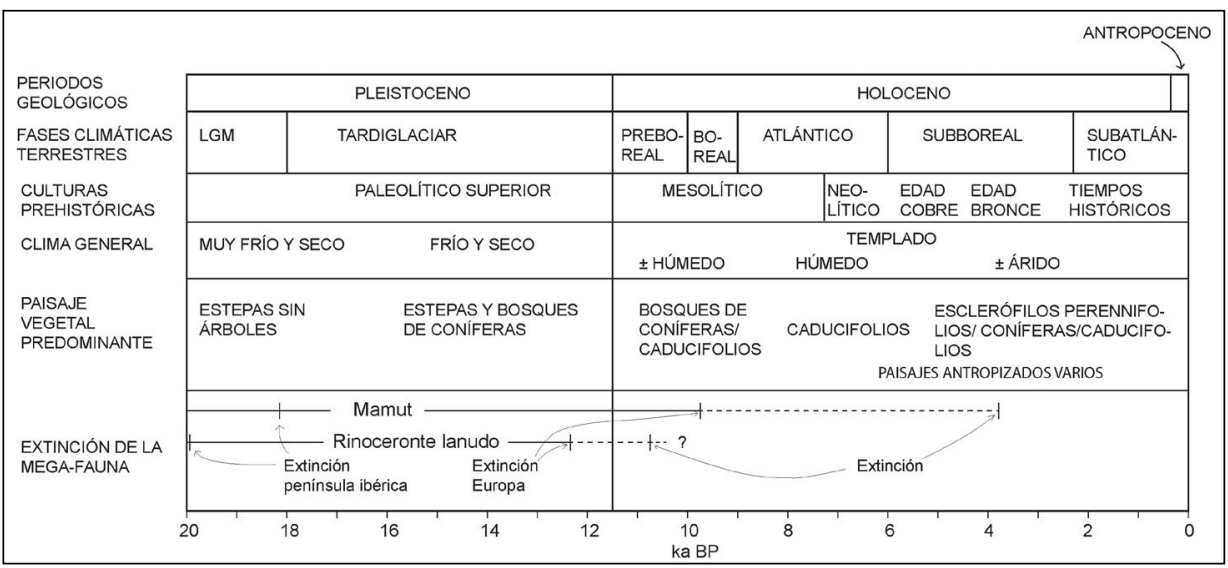

Figura 2. Esquema cronológico de los periodos geológicos, las fases climáticas terrestres, las culturas prehistóricas, el clima general, el paisaje vegetal dominante y la extinción de la megafauna en los últimos 20.000 años, principalmente en la península Ibérica. Realizado a partir de Carrión (2002), Stuart (2005), González Sampériz et al. (2008 y 2017), Álvarez-Lao y García (2010), Carrión et al. (2010 a), Pérez Obiol et al. (2011), Cortés Sánchez et al. (2012), Markova et al. (2013), Rillardon y Brugal (2014), Pérez-Díaz et al. (2015), Stuart (2015) y Dias et al. (2016).

\subsection{Holoceno: recuperación forestal y sus consecuencias}

La suavización del clima a final del Pleistoceno e inicio del Holoceno produjo una gran expansión de los bosques en Europa (Huntley y Birks, 1983; Berglund et al., 1996, Guthrie, 2001; Carrión et al., 2010a; Allen et al., 2010; Collins et al., 2012; Huntley et al., 2013; Aranbarri et al., 2014) que llegó a ser máxima hacia el 
Holoceno medio (Roberts et al., 2018). Firbas (1949) e Iversen (1960) plantearon que las zonas templadas de Europa estarían cubiertas por densos bosques mixtos de caducifolios durante el máximo climático del Holoceno, hace unos 6000 años. Esta hipótesis se ha mantenido sin cuestionamientos importantes hasta el final del siglo XX. Sin embargo, en las dos últimas décadas se está produciendo un amplio debate sobre el grado de "apertura" que deberían tener los bosques de los paisajes naturales europeos sin la intervención humana, incentivado por la necesidad de disponer de modelos para la gestión y restauración de los espacios naturales (Svenning, 2002; Birks, 2005). Vera (2000) propuso que los bosques naturales estarían modelados por los grandes herbívoros que mantendrían paisajes abiertos con un mosaico de pasto, arbolado y matorral. Argumentaba que los robles y avellanos, abundantes en los registros polínicos analizados de las zonas templadas europeas, indicarían bosques abiertos ya que esas especies no pueden germinar en ambientes muy sombríos. Varios estudios han explorado las dos hipótesis en el centro y norte de Europa y han encontrado más soporte para la clásica de Firbas e Iversen que para la de Vera (Mitchell, 2005; Whitehouse y Smith 2010; Sandom et al., 2014b). Por el contrario, los estudios desarrollados principalmente en el sur o el este de Europa sugieren la existencia de espacios abiertos durante todo el Holoceno, ya sea por efectos del clima, del suelo o de los incendios frecuentes (Ellenberg, 1988; Mason, 2000; González-Sampériz et al., 2008; Kunes et al., 2015; Pokorny et al., 2015). En la península Ibérica, el paisaje vegetal durante el Holoceno medio estuvo dominado por diversos bosques caducifolios de clima templado (Ramil-Rego et al., 1998; Benito Garzón et al., 2007; Carrión et al., 2010a, 2010b), aunque también persistieron algunas zonas de estepas y bosques poco densos (González-Sampériz et al., 2008).

Entre los factores ecológicos que determinaban la densidad de los bosques, el efecto de la megafauna sería muy importante, incluso en las condiciones favorables para el desarrollo vegetal de los interglaciares (Bakker et al., 2016), como se observa en la actualidad con la escasa megafauna que aún persiste (Owen-Smith, 1988; Asner y Levick, 2012; Staver y Bond, 2014). Por ello, cabe esperar que los bosques durante los primeros milenios del Holoceno fueran más densos y extensos que en los periodos equivalentes de los interglaciares anteriores ya que al inicio del Holoceno la megafauna estaba prácticamente extinta (Bakker et al., 2016; Gill, 2014) (Fig. 2). Por el contrario, en los interglaciares previos al Holoceno la megafauna fue abundante y diversa (Agustí y Antón, 2002; Magri y Palombo, 2013). El debate sobre la extensión y densidad de los bosques y de los espacios abiertos en el Holoceno y en los anteriores interglaciares se ha basado, por ejemplo, en la comparación de análisis de polen fósil (West et al., 1995; Keen et al., 1999), las proporciones de moluscos y escarabajos de ambientes abiertos y forestales (Ponel, 1995; Alexander, 2005; Whitehouse y Smith, 2010; Sandom et al., 2014b), los porcentajes de isótopos estables en tejidos perdurables de vertebrados herbívoros (Drucker et al., 2003, 2008; Bocherens et al., 2015; Hofman-Kaminska et al., 2018), la presencia de esporas en el suelo de hongos coprófilos del estiércol de los grandes herbívoros (Bakker et al., 2016), etc. Distintos trabajos sostienen que los bosques de la primera mitad del Holoceno en Europa eran densos o muy densos (Whitehouse, 1997; Dinnin y Sadler, 1999; Bradshaw et al., 2003; Mitchell, 2005; Whitehouse y Smith, 
2010; Sandom et al., 2014b), aunque abundaban algunas especies que regeneran mal en ambientes muy sombríos, como Quercus spp., Corylus avellana y Pinus sylvestris (Vera, 2000; Svenning, 2002). Sin embargo, los bosques de los interglaciares anteriores al Holoceno estarían en mosaico con los pastos (ver Svenning, 2002 para una revisión del tema), aunque algunos autores aprecian fases de bosques caducifolios densos (Kunes et al., 2011). El debate científico abierto sobre esta cuestión no permite extraer conclusiones definitivas (Gill, 2014).

Otro factor muy importante en la discusión anterior es el fuego. Se suele interpretar que los incendios ocurridos en Europa a partir del Neolítico dependieron en gran medida de la actividad humana y que los anteriores dependieron de factores naturales, aunque sin excluir totalmente los de origen antrópico (Gil Romera et al., 2010; Vannière et al., 2011; Morales-Molino et al., 2013). Sin embargo, los homininos comenzaron a manejar el fuego desde tiempos muy remotos y, sin duda, a comienzo del Holoceno los humanos ya eran expertos en su uso (Marlon et al., 2013) por lo que algunos autores han inferido la importancia del fuego antrópico antes del Neolítico (Mason, 2000; Attwell et al., 2015; Lawson et al., 2013) e incluso durante el LGM (Kaplan et al., 2016), que presenta la mínima frecuencia de incendios de los últimos 21.000 años por la escasez de combustible (Power et al., 2008). Sin embargo, el progreso en esta dirección es muy comprometido por la gran dificultad de distinguir la causa del fuego en los registros paleo-ecológicos anteriores al Neolítico (Vannière et al., 2011; Kaal et al., 2011; Marlon et al., 2013; García-Ruiz et al., 2016).

Los acusados cambios de paisaje vegetal que ocurrieron en Europa entre el último episodio glacial y el Holoceno tuvieron importantes consecuencias ecológicas. Una de ellas, derivada de la gran expansión forestal que se produjo desde el final del Pleistoceno hasta el Neolítico, pudo conducir a carencias alimentarias en muchas poblaciones humanas que en el Pleistoceno habían tenido su principal sustento en los grandes herbívoros pastadores (Niven, 2007; Richards, 2009). Son muy numerosos los trabajos que describen un importante cambio de alimentación entre el final del Pleistoceno y el inicio del Holoceno en las poblaciones humanas europeas, que tendieron a quedar relegadas a las zonas costeras ricas en recursos marinos (Schulting, 2011). En los yacimientos estudiados se observa una importante disminución de los restos de ungulados y un aumento considerable de los vegetales, pequeños vertebrados terrestres, peces y recursos marinos (Richards, 2002; Marín-Arroyo, 2013; Rillardon y Brugal, 2014; Zaatari y Hublin, 2014; Fernández-López de Pablo y Gabriel, 2016; Dias et al., 2016). Incluso se ha descrito que los pobladores neolíticos ocuparon amplios territorios en el sur de la península Ibérica que, al parecer, ya habían abandonado los anteriores pobladores mesolíticos (Cortés Sánchez et al., 2012). Sin embargo, en otras zonas de Europa se ha documentado una coexistencia bastante prolongada de las dos culturas hasta la desaparición de los cazadores-recolectores (González-Fortes et al., 2017; Innes et al., 2013). Es interesante observar que este cambio de la alimentación humana pudo ocurrir antes en la península Ibérica que en el centro y norte de Europa (Dias et al., 2016), seguramente porque la expansión forestal fue anterior en el sur del continente. 


\subsection{Neolítico}

El Neolítico en Europa se inició con la adopción de la agricultura y la ganadería y supuso un incremento importante de los incendios desbrozadores de origen antrópico (Vannière et al., 2011; Rius et al., 2011; Marlon et al., 2013; Morales-Molino y García-Antón, 2014). Ahora ya no se trataba de frenar el avance del bosque y crear algunos claros forestales sino de modificar el paisaje para expandir campos de cultivo y pastos (Kaal et al., 2011; Innes et al., 2013). Para este fin se requirió un uso intensivo del fuego, que fue en aumento a medida que las poblaciones humanas crecieron y necesitaron ocupar territorios más extensos y explotarlos con mayor intensidad. En la península Ibérica se observan notables asincronías entre los regímenes de incendios de localidades del norte y del sur. En los registros palo-ecológicos, a partir de los 40003500 años BP se detectan frecuentes incendios de origen claramente antrópico, que aumentan de manera generalizada en toda la península después de los 2800 años BP (Vannière et al., 2011).

El uso del fuego como agente desbrozador sin duda tuvo importantes y crecientes consecuencias en el paisaje por reducción drástica del bosque y expansión de los pastos y campos de cultivo (Kaal et al., 2011; Morales-Molino y García-Antón, 2014; García-Ruiz et al., 2016). La reiteración en el uso del fuego para someter árboles y arbustos ocasionó la degradación del territorio por la pérdida de fertilidad y suelo y la expansión de matorral muy poco productivo (Kaal et al., 2011; Morales-Molino y García-Antón, 2014). Esta actividad ha dejado numerosas huellas geomorfológicas en el paisaje que se reconocen en la actualidad (García-Ruiz et al., 2016).

La degradación antrópica de los bosques y el desarrollo de la ganadería facilitaron la expansión de los pastos. La flora de los pastos actuales procede de restos de pastos primarios y de poblaciones acantonadas en refugios a salvo del dominio boscoso prevalente en la mayor parte de Europa al inicio del Neolítico (Pott, 1995; Bredenkamp et al., 2002). Sin embargo, en el centro y este de Europa parece que persistieron algunos retazos de pastos primarios durante todo el Holoceno (Hejcman et al., 2013; Kunes et al., 2015; Pokorny et al., 2015), al igual que debió ocurrir en otros territorios, como en algunas zonas del sur de la península Ibérica (Bredenkamp et al., 2002).

\subsection{Edad Media}

A partir del Neolítico continuó y se acrecentó la transformación del paisaje para ampliar cultivos, pastos e infraestructuras. En el registro paleo-ecológico se aprecian periodos con distinta degradación de la vegetación arbórea dominante siguiendo el curso de los acontecimientos históricos de cada región peninsular (Carrión et al., 2007; López Sáez et al., 2009, 2014; Cunill et al., 2013; Morales-Molino y García-Antón, 2014; Aranbarri et al., 2014). Este proceso parece que culminó durante la Edad Media en bastantes territorios peninsulares (Montserrat Martí, 1992; López Sáez et al., 2014; Cunill et al., 2013; García Ruiz et al., 2016). En este periodo se ajustaron los mecanismos de explotación agropecuaria a las com- 
plejas peculiaridades orográficas y climáticas de la península Ibérica (López Sáez et al., 2009) y se potenciaron tres tipos de movimiento estacional de los rebaños domésticos: interregional (trashumancia), intercomarcal (transterminancia) y local para adaptar el pastoreo a la fuerte estacionalidad climática y a la complementariedad de los diferentes territorios peninsulares. El alto grado de sofisticación que alcanzó el pastoralismo en la Edad Media requirió la creación de varias organizaciones de ganaderos, como el Honrado Concejo de la Mesta de Castilla y León y la Casa de Ganaderos de Zaragoza, entre otras. Los ganaderos consiguieron mantener grandes rebaños mediante movimientos estacionales que evitaban el aporte de forrajes adicionales. Esta optimización del aprovechamiento limitaba sustancialmente la acumulación de materia vegetal seca. El pastoreo y la extracción de leñas contuvieron la invasión de los arbustos en los pastos y, lo más importante, conformaron y conservaron unos agro-ecosistemas de gran valor natural con extraordinaria biodiversidad (Zorita, 2001). El sistema trashumante inició un fuerte crecimiento a partir del siglo XV y alcanzó su máximo desarrollo en el siglo XVIII gracias al comercio internacional de lanas finas, pero a final de este mismo siglo comenzó un declive que culminó en 1836 con la abolición de la Mesta (Pérez Romero, 2007, 2016). Las causas del hundimiento de los sistemas trashumante y transterminante fueron diversas: los procesos desamortizadores de Mendizábal y Madoz, la pérdida del monopolio de la producción de lanas finas, el incremento demográfico que se produjo en el siglo XIX, el abandono de las prácticas zootécnicas dirigidas a producir lana de calidad en aras a aumentar la producción, el aumento de los costes de los pastos de invierno, etc. (Zorita, 2001; Collantes Gutiérrez, 2003; Pérez Romero 2007). Tras la abolición de la Mesta las trashumancias quedaron muy reducidas en la península Ibérica pero se mantuvieron o incluso se potenciaron los movimientos locales (Zorita, 2001). Persistió una importante ganadería extensiva hasta la década de los 60 del siglo XX, cuando la producción de carne pasó a depender en su mayor parte de explotaciones intensivas que ya no estaban ligadas a la producción local de los pastos (Collantes Gutiérrez, 2003). En dos decenios se derrumbaron los agro-ecosistemas milenarios de la península Ibérica que estaban vertebrados por un intenso uso pastoral (Zorita, 2001).

La organización de la ganadería iniciada en el Neolítico y con máximo desarrollo en los sistemas trashumantes de los siglos XIII-XVIII, tuvo importantes consecuencias ecológicas en la península Ibérica (Ruiz y Ruiz, 1986; Zorita, 2001). Restableció el efecto de los herbívoros pastadores por medio de la domesticación y favoreció la expansión de las comunidades herbáceas de ambientes abiertos, lo que contribuyó a equilibrar en el paisaje las áreas de bosque-matorral y los pastos herbáceos. Sin duda, este fenómeno favoreció la expansión de numerosas plantas y animales de lugares abiertos que habrían quedado muy acantonadas en el periodo de máxima expansión forestal del Holoceno medio, facilitando la conservación de la extraordinaria biodiversidad de la península Ibérica (Zorita, 2001; Montserrat et al., 2017). El esquema de la Figura 3 resume la diversidad de especies y ambientes que habitan en Aragón, como ejemplo de un territorio significativo de la península Ibérica. 


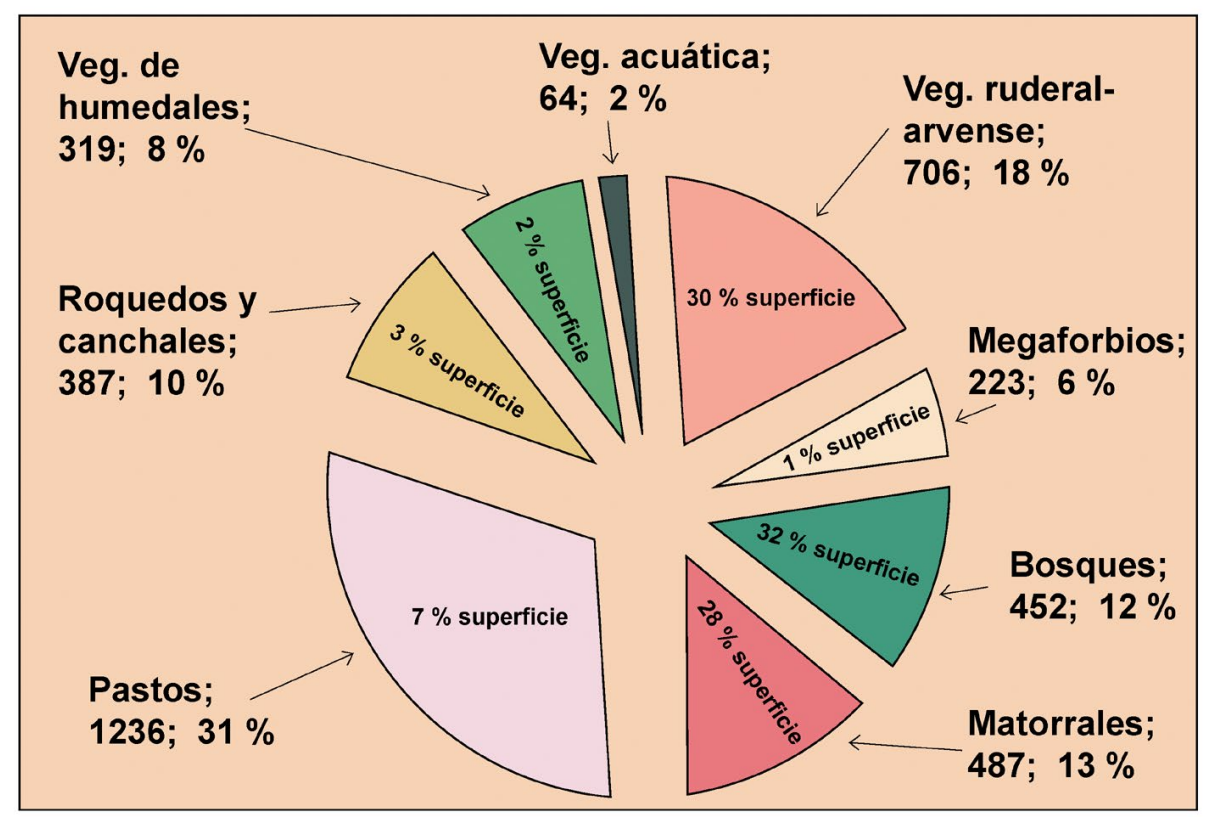

Figura 3. Distribución aproximada de la diversidad de especies de plantas vasculares en Aragón según los tipos de vegetación y los porcentajes de superficie que ocupan. La diversidad florística de Aragón se puede considerar que es similar a la de la flora Ibérica. Elaboración propia a partir de los datos del Atlas de la flora de Aragón (http://floragon.ipe.csic.es/index.php).

\subsection{El paisaje actual}

La liquidación del Antiguo Régimen (abolición de la Mesta en 1836) supuso la limitación de los movimientos de los rebaños a zonas más locales y la necesidad de tener que complementar su alimentación durante algunos meses del año con piensos producidos localmente y forrajes almacenados. Por primera vez la ganadería comenzó a depender parcialmente de la agricultura. Esta tendencia ha ido en aumento, muy especialmente a partir de la segunda mitad del siglo XX. A comienzo de la década de 1960 se inició un cambio drástico del país con la apertura de la economía al comercio y la financiación internacional, la industrialización, el crecimiento urbano y el progresivo abandono del campo (Zorita, 2001). La ganadería extensiva inició un fuerte declive, se abandonaron muchos pastos y se potenció la ganadería intensiva con una imagen de progreso. La alimentación del ganado pasó a depender casi exclusivamente de los productos agrícolas, principalmente de los piensos con maíz y soja, a la vez que prácticamente desaparecía el ganado equino y se sustituían las razas autóctonas más rústicas por otras más selectas importadas para aumentar la producción. Por su parte, la agricultura se intensificó en los llanos y se abandonó también en gran parte la de la montaña, haciéndose cada vez más dependiente de los combustibles fósiles, los fertilizantes y productos fitosanitarios industriales. La agricultura ha sido desde sus inicios una actividad de efecto ambiental degradante ya que necesita roturar el suelo 
para aliviar su compactación, controlar las malas hierbas y facilitar la siembra, incorporar abonos y promover la infiltración del agua. En este proceso los niveles internos del suelo se exponen a la intemperie y a la luz solar directa con lo que se oxida la materia orgánica y se esteriliza una parte de su flora microbiana. Es decir, se reducen dos de los principales factores de la fertilidad natural. Ello determina que los suelos agrícolas sean más proclives a la erosión física de las partículas y a la erosión química de algunos nutrientes que los de los pastos. Además, en el último siglo se han intensificado los problemas ecológicos asociados a la agricultura por la mecanización de las labores agrícolas y la aplicación generalizada de abonos y pesticidas químicos (Zalidis et al., 2002; Conant et al., 2007; Stoate et al., 2009).

Como no podía ser de otro modo, los paisajes vegetales de la península también experimentaron un fuerte cambio a partir de la década de 1960. Las inmensas superficies de pastos herbáceos apenas arboladas que anteriormente cubrían amplios territorios, se transformaron con rapidez por la repoblación forestal y la expansión de montes bajos y matorrales muy densos. La escasa presión ganadera y la radical prohibición de realizar incendios desbrozadores aceleraron sustancialmente este proceso de reforestación. En las llanuras los cambios no fueron menores y muchos paisajes devinieron en cultivos, núcleos urbanos y sus infraestructuras con apenas algunos retazos de vegetación ruderal como único vestigio de la naturaleza.

En climas como el actual, adecuados para el crecimiento forestal, la colonización de los pastos por arbustos y árboles (proceso que se conoce como matorralización) suele ser bastante rápida tras el cese del fuego y la disminución del pastoreo, incluso en las condiciones más duras de las montañas (Montserrat et al., 2017).

En los últimos años se ha propuesto la reintroducción de fauna silvestre de otros territorios para restituir la extinta (rewilding), tratando de restablecer los procesos naturales con la mínima intervención humana (Navarro y Pereira, 2012; Carey, 2016).

\section{Discusión}

La bibliografía disponible no permite avalar ni rechazar la hipótesis de una expansión forestal extensiva y masiva en la primera mitad del Holoceno por déficit de grandes herbívoros. También restan en la incertidumbre la abundancia de grandes herbívoros en el Pleistoceno y sus efectos en la vegetación, las causas de la extinción de los grandes vertebrados a final de ese período, las variaciones de extensión y densidad de los bosques entre el Holoceno y los interglaciares anteriores, la existencia de evidencias claras en los registros paleo-ecológicos de las perturbaciones originadas por los humanos para limitar la extensión forestal y favorecer a los herbívoros pastadores, etc. Varios de estos temas sobre los que pivota nuestra hipótesis se debaten actualmente y dan lugar a interpretaciones contrapuestas. Comentamos a continuación los aspectos que nos parecen más relevantes, proponiendo algunas ideas para su interpretación y discutiendo las limitaciones de la información paleo-ecológica publicada para responder a preguntas tan generales como las que aquí planteamos.

\subsection{Efecto de los grandes herbívoros sobre la vegetación}

La reconstrucción de los paisajes vegetales pretéritos suele apoyarse en las variaciones del clima derivadas de modelos y, en menor medida, en los regímenes de perturbación, 
entre los que destacan los debidos al fuego (Bond et al., 2005). Las reconstrucciones realizadas del LGM no suelen considerar el efecto de los herbívoros de tallas grande y media sobre la vegetación, aunque algunos autores sí consideran su importancia (Zimov et al., 1995; Coope, 2000; Rule et al., 2012; Gill et al., 2012, 2013; Gill, 2014, etc.). Con estos autores, defendemos el destacado efecto de estos animales por los siguientes motivos:

1. Los grandes herbívoros, capaces de consumir plantas leñosas poco nutritivas (Demment y Van Soest, 1985; Wing y Tiffney, 1987) junto a otros herbívoros de dieta más selectiva, habrían generado pastos muy productivos que permitirían la subsistencia de numerosos vertebrados de distintas tallas (Montserrat Recoder, 1964; Owen-Smith, 1987). Un proceso similar se ha descrito en África por el pastoreo combinado de elefantes, rinocerontes e hipopótamos que ha producido los pastos que sustentan la mayor biomasa animal medida en ambientes naturales (Owen-Smith, 1987). Aunque no es fácil calcular el tamaño de las poblaciones de los herbívoros del Pleistoceno a partir de los restos fósiles (Bradshaw et al., 2003), se ha podido estimar que en las estepas de Alaska y del NE de Siberia durante el último episodio glacial la densidad de los grandes herbívoros era de 88 y $105 \mathrm{Kg}_{\text {.ha }}{ }^{-1}$, respectivamente (Mann et al., 2013; Zimov et al., 2012). Es decir, la productividad vegetal y la biomasa animal de la estepa glacial, en un lugar tan frío e inhóspito como es el norte de Siberia, sería similar a la de las sabanas africanas actuales (Zimov et al., 2012).

2. Durante el LGM debieron ocurrir importantes migraciones de herbívoros de tamaños grande y medio forzadas por la fuerte estacionalidad del clima. Cabe suponer que se producirían migraciones de grandes rebaños hacia el centro y el norte de Europa en primavera y que regresarían al sur de Europa en otoño. Los análisis de los isótopos de estroncio, oxígeno y carbono en la dentina proporcionan información sobre la variación de ambientes, climas y alimentos que experimentaron los individuos analizados durante el periodo de formación de sus dientes (Julien et al., 2012). Con este método se ha podido inferir que los renos, mamuts y mastodontes podían realizar largas migraciones estacionales y que los bisontes y caballos tendían a ser más sedentarios (Hoppe y Koch, 2007; Pellegrini et al., 2008; Britton et al., 2011; Julien et al., 2012). Sin embargo, también se ha estimado una escasa movilidad en algunos casos analizados de especies potencialmente migratorias (Hoppe y Koch, 2007). Las migraciones de renos debieron ser muy regulares y constituyeron un recurso esencial para las culturas paleolíticas en los periodos más fríos (Niven 2007).

Los movimientos estacionales de grandes herbívoros supondrían la acumulación de muchos individuos en determinados periodos del año como puede observarse actualmente en las grandes estepas centroafricanas. Y como allí, en estos periodos de intenso pastoreo (consumo, pisoteo y aporte de excrementos) la vegetación leñosa más extendida en el LGM se vería particularmente dañada, favoreciendo el dominio del pasto herbáceo (Bond, 2010). Tal como se observa hoy, durante los periodos de fuerte estrés climático (principalmente en invierno), los herbívoros se verían forzados a consumir todo tipo de plantas, incluyendo 
las leñosas capaces de resistir las condiciones climáticas del LGM (especies con tendencia a ser tolerantes al estrés). Muchas de estas plantas no podrían recuperarse de tales perturbaciones y, por tanto, cabe suponer que quedarían relegadas a los lugares menos accesibles, lo que podría explicar la ausencia de árboles en extensas zonas de Europa durante los interestadios relativamente templados del último ciclo glacial (Kolstrup, 1990).

El movimiento estacional de los herbívoros con intensa carga temporal habría favorecido un aprovechamiento óptimo de la vegetación que propiciaría un mosaico de pastos en distintas condiciones de clima, topografía, sustrato y fertilidad del suelo. Esta heterogeneidad vegetal y animal mantendría un sistema de alta producción primaria y consumo muy intenso (Zimov et al., 2012; Zhu et al., 2018).

3. Las poblaciones humanas que persistieron en Europa durante el LGM debieron cazar profusamente para abastecerse de carne, grasa y pieles esenciales para su supervivencia (Flodin, 1999). Cabe suponer que dicho aprovisionamiento se beneficiaría de las migraciones de final de verano y principio de otoño. La caza sostenida y la adquisición progresiva de nuevas técnicas conducirían a una presión cinegética cada vez mayor. Aunque no existe acuerdo entre los expertos, numerosos autores coinciden en que ésta fue una causa importante de la extinción de la megafauna en Europa a final del Pleistoceno (Stuart, 2015).

\subsection{Extinción de la megafauna en Europa}

Durante todo el Pleistoceno, plantas y animales adaptados a los climas fríos y secos de ambientes abiertos se extendieron por inmensas superficies durante los episodios glaciales y retrocedieron a enclaves de clima adecuado, de extensión mucho más reducida, durante los interglaciares. Lo contrario ocurría con las especies dominantes durante los interglaciares, que quedaban reducidas a modestos enclaves en los lugares más abrigados del sur de Eurasia durante los episodios glaciales. De este modo, muchas especies persistieron y evolucionaron en Eurasia durante el Pleistoceno, experimentando diversas expansiones y contracciones de sus áreas de distribución asociadas a los sucesivos ciclos glaciales (Markova et al., 2013). Sin embargo, en el último episodio glacial se extinguió toda la megafauna de Europa que había existido en este continente durante millones de años (Agustí y Antón, 2002; Stuart, 2005). Esta anomalía puede achacarse a que el clima del último ciclo glacial fuera extraordinariamente desfavorable para un gran número de mamíferos de cierta talla (Graham y Lundelius, 1984; Guthrie, 1984) o a que la presión antrópica resultara decisiva para su extinción (Martin, 1967; Martin y Steadman, 1999; Gill et al., 2009; Sandom et al., 2014a). Es lógico suponer que incluso una presión cinegética moderada en los periodos de regresión y reducción a los refugios, cuando la megafauna era más vulnerable, resultara fatal para su supervivencia (Stuart, 2005; Zuo et al., 2013). Antes de la aparición del género Homo en Eurasia, la extinción de mamíferos por fuertes cambios ambientales fue frecuente y daba lugar a la evolución o inmigración de especies equivalentes ecológicamente a las desaparecidas que ocupaban sus 
nichos vacíos (Agustí y Antón, 2002; Stuart, 2015). Muy posiblemente, la ausencia en el Holoceno de esta sustitución se debió a causas exclusivamente antrópicas. Sin Homo es muy probable que la megafauna persistiera hoy en Europa y, a falta de depredadores tan efectivos, podría mantener poblaciones densas y ejercer un importante efecto sobre la vegetación (Zimov et al., 1995; Johnson, 2009; Bakker et al., 2016), como ocurre actualmente en los escasos territorios donde los mega-herbívoros pueden vivir sin riesgos importantes de predación humana (Owen-Smith, 1987, 1988; Scholes y Archer, 1997; Asner et al., 2012, 2016).

\subsection{Expansión forestal en el Holoceno}

Nuestra hipótesis supone que en la primera mitad del Holoceno las plantas y animales de lugares abiertos, que dominaron los paisajes de los periodos fríos del Pleistoceno, quedaron relegados en gran parte de Europa por la expansión masiva del bosque, efecto que ha sido señalado por distintos autores (Ellenberg, 1988; Pott, 1995; Ricánková et al., 2018). Estos organismos sólo han podido persistir en zonas rocosas o en sustratos poco favorables para el bosque (roquedos, yesos, serpentinas, zonas salobres, etc.). Allí suelen refugiarse poblaciones relativamente pequeñas que pueden quedar bastante aisladas. La flora Ibérica contiene una gran mayoría de especies de lugares abiertos (Castroviejo et al., 1986, 2015) (Fig. 3), lo que refleja perfectamente las condiciones ambientales del Cuaternario con predominio de los episodios glaciales sobre los interglaciares (Kukla, 2005). Si consideramos las plantas herbáceas o matas de lugares abiertos en clima estresante, seguramente las más compatibles con los ambientes fríos y secos de los periodos glaciales, encontramos que son numerosas en el sur de la península (Blanca et al., 2011; Valdés et al., 1987), sin llegar a ser raras en el norte peninsular. Por ejemplo, Scabiosa graminifolia ocupa en el Pirineo enclaves abruptos del piso subalpino pero desciende a $1000 \mathrm{~m}$ en zonas rocosas de Egea de Turbón (Huesca), donde también persisten Erinacea anthyllis y Euphorbia minuta, otras dos rarezas en el Pirineo aragonés (Montserrat Martí, 1986). En Álava S. graminifolia aparece en crestones venteados y secos de baja altitud (900-1300 m), en el sistema ibérico a unos 1700 m (Gómez García et al., 2005; Aseginolaza et al., 1984; Uribe-Echebarría y Zorrakin, 2004) y en las sierras prelitorales catalanas en altitudes moderadas (ORCA). Entre otros ejemplos, Plantago argentea, Jurinea humilis y J. pinnata son especies raras en Aragón cuyas poblaciones se refugian en enclaves pedregosos que seguramente no se llegaron a cubrir por bosques durante el Holoceno (Gómez García et al., 2005). Para estas plantas, posiblemente mucho más extendidas en los periodos fríos y secos del Pleistoceno, la sombra de los árboles es mucho más perniciosa que cualquiera de los cambios climáticos acaecidos en el Holoceno. Este fenómeno de arrinconamiento por la gran expansión forestal de ciertos grupos florísticos a los refugios donde ahora persisten podría explicar la distribución fragmentada y la rareza de muchas especies herbáceas perennes y leñosas, algunas amenazadas de desaparición en la península Ibérica. Probablemente, la apertura del bosque en el Neolítico y el desarrollo de la ganadería no permitieron su expansión posterior a partir de los refugios, debido a que el clima del interglaciar (Holoceno) era poco adecuado para 
la expansión de estas especies, muy extendidas en los periodos glaciales y refugiadas en los interglaciares.

Las plantas de los pastos son más tolerantes a la perturbación que las tolerantes al estrés que acabamos de comentar (Grime et al., 2007) pero tampoco soportan la sombra del bosque y, por tanto, también sufrirían una importante regresión en los primeros milenios del Holoceno en Europa (Pott, 1995; Bredenkamp et al., 2002; Pokorny et al., 2015). Sin embargo, las especies de los pastos pueden refugiarse en orlas y claros forestales generados por los animales, el viento, los procesos geomorfológicos, etc. (Pott, 1995). La destrucción del bosque que se produjo en el Neolítico permitió su expansión fomentada por la ganadería. Los nuevos pastos serían parecidos a los del Pleistoceno aunque con las diferencias impuestas por el clima más benigno y los distintos herbívoros (Bredenkamp et al., 2002).

Entre los animales también se encuentran casos de regresión y extinción por la expansión forestal de final del Pleistoceno y principio del Holoceno. Por ejemplo, la marmota, que a principio del Holoceno se extinguió del Pirineo donde había persistido durante todo el Pleistoceno, se reintrodujo con mucho éxito en el siglo XX. En la actualidad la mayoría de colonias se encuentran entre 1800 y 2400 m, por encima del límite del bosque hasta donde la escasez de vegetación ya no permite su supervivencia. El notable ascenso del límite del bosque que se produjo en el Pirineo durante los primeros milenios del Holoceno (se estima en unos $400 \mathrm{~m}$ superior al límite actual, Cunill et al., 2012) limitó extraordinariamente el hábitat de la marmota y debió conducirla a la extinción. La expansión de los pastos después del Neolítico restituyó su hábitat y permitió la rápida recolonización que se produjo recientemente (Herrero et al., 1994). Los caballos (Equus ferus) fueron una presa importante para los humanos europeos del último episodio glacial. A comienzo del Holoceno se produjo una gran regresión de sus poblaciones debida a la pérdida de los hábitats abiertos. Tras la degradación de los bosques en el Neolítico, las poblaciones de caballo silvestre se extendieron de nuevo por Europa (Sommer et al., 2011).

Homo sapiens también debió padecer la expansión de los bosques ya que nuestra especie evolucionó en espacios abiertos de África (Agustí y Antón, 2011) y la transformación del paisaje de estepas y tundras con escaso arbolado en uno forestal no le debió ser nada propicia. Es razonable suponer que tratara de limitarla mediante el fuego, que sería el procedimiento más eficaz para recuperar paisajes abiertos con pastos y sus plantas más nutritivas.

\subsection{Controversia sobre las causas del fuego a comienzos del Holoceno}

En los registros paleo-ecológicos europeos son numerosos los estudios que observan incendios frecuentes entre el final del Tardiglacial y el comienzo del Holoceno (Power et al., 2008; Vannière et al., 2008; Rius et al., 2011; Cunill et al., 2012, 2013; Morales-Molino y García-Antón, 2014; Gil-Romera et al., 2014; Lehndorff et al., 2015), aunque con notables diferencias en distintos territorios (Gil-Romera et al., 2010; Kaal et al., 2011; Marlon et al., 2013). En general, estos incendios se 
interpretan como la respuesta natural a la acumulación de biomasa, la elevada insolación estival y la sequedad del clima (Carrión, 2002; Vannière et al., 2008, 2011; Morales-Molino et al., 2013). Sin embargo, algunos autores han manifestado dudas sobre las causas naturales del fuego de la primera mitad del Holoceno. Por ejemplo, Lawson et al. (2013) en un estudio realizado en Ioannina (Grecia) interpretan que la mayor parte de los incendios en los primeros 5000 años del Holoceno era de origen antrópico, lo que marcaba una importante diferencia con el periodo equivalente del anterior interglaciar. Cabe suponer que los incendios de origen antrópico de los primeros milenios del Holoceno servirían para extender los claros forestales y facilitar así la recolección y la caza. Este esfuerzo sería una batalla perdida ya que los bosques se recuperarían con rapidez de los incendios (Gómez Gutiérrez et al., 1988; Bartolomé et al., 2000; Gómez García et al., en revisión). Además, la reiteración del fuego degradaría la vegetación por pérdida de suelo y fertilidad y por sustitución de las especies dominantes por geófitos de escaso valor pastoral y arbustos pirófitos poco productivos como los brezos, jaras, leguminosas espinosas, etc. (Tinner et al., 2005; Gil-Romera et al., 2010; Kaal et al., 2011). Las manadas de herbívoros pastadores seguirían disminuyendo y, con este declive, también disminuiría la población humana. La baja densidad de población durante los siguientes milenios (Valdeyron, 2008) y la limitada capacidad de combustión de los bosques de caducifolios, dificultarían el mantenimiento de los paisajes abiertos.

En los registros paleo-ecológicos difícilmente se puede asegurar la causa de los incendios, muy especialmente en los anteriores al Neolítico (Vannière et al., 2011; Kaal et al., 2011, Marlon et al., 2013; García-Ruiz et al., 2016). Además, la interpretación del origen natural de los incendios se basa en la estrecha relación que se observa entre el fuego con la dinámica del clima, el tipo de vegetación y la acumulación de biomasa inflamable. No obstante, si su origen fuera antrópico también cabría esperar relaciones semejantes, ya que no tiene sentido quemar los montes si no se acumula suficiente biomasa combustible o si el clima es demasiado húmedo. Por lo tanto, debemos esperar que las respuestas observadas en los registros paleo-ecológicos sean muy similares tanto en los incendios producidos por causas naturales como antrópicas.

\subsection{Expansión forestal de las últimas décadas en la península Ibérica}

El abandono reciente de los pastos en grandes extensiones de la península Ibérica está favoreciendo la colonización de arbustos poco palatables (matorralización) que evidencian la evolución de praderías y campos agrícolas abandonados hacia comunidades forestales. Esta expansión forestal guarda cierto paralelismo con la que se produjo en los primeros milenios del Holoceno. Ambas ocurrieron con una importante deficiencia de grandes herbívoros, aunque la primera se asoció a un importante cambio del clima y la segunda no.

Los paisajes que consideramos más naturales y diversos de la península Ibérica se originaron tras siglos de pastoreo (Zorita, 2001) y, anteriormente, tras muchos milenios de pastoreo por un nutrido y diverso grupo de herbívoros. Esta riqueza biológica 
muy productiva desaparece con rapidez tras el derrumbe de la vida rural tradicional, al tiempo que los pastos se cubren de plantas leñosas, generalmente de crecimiento lento (pinos, bojes, enebros, avellanos, rosáceas y leguminosas espinosas, etc.), cuando no se destruyen por la repoblación forestal con pinos, eucaliptos o quercíneas. La mayoría de estas plantas producen hojarasca poco nutritiva que genera suelos de escasa fertilidad, lo que dificulta o impide la recuperación del pasto (Aerts y Chapin, 2000; Montserrat et al., 2017). Sin duda, esta nueva expansión forestal empobrecerá los ecosistemas naturales, mientras que las plantas y los animales propios de los espacios abiertos más productivos verán comprometida su persistencia con la consiguiente pérdida de diversidad.

La solución fácil para el desbroce de los pastos que se matorralizan es el uso del fuego pero, tras la quema, la mayoría de las especies matorralizadoras recuperan sus poblaciones vigorosa y rápidamente por rebrote o germinación (Gómez García et al., en revisión). Cabe suponer que si persistieran los mega-herbívoros ramoneadores y pastadores, que se extinguieron a final del Pleistoceno en la península Ibérica (proboscídeos y rinocerontes), no haría falta el fuego desbrozador ya que estos animales arrancarían y aplastarían numerosas plantas leñosas y, además, a causa de su gran tamaño, podrían consumir mucha más biomasa leñosa que los herbívoros domésticos (Demment y Van Soest, 1985; Wing y Tiffney, 1987). La actividad de estos grandes animales crearía amplios claros en bosques y matorrales que los ungulados pastadores podrían transformar en pastos (Bond, 2010), lo que generaría paisajes en mosaico muy diversificados y productivos (Sandom et al., 2014b; Bakker et al., 2016). Por lo tanto, la introducción de elefantes y rinocerontes en nuestros montes para facilitar el desbroce, como debieron hacer los proboscídeos y rinocerontes extintos, podría ser una solución efectiva. Sin embargo, el manejo de estos animales parece inviable actualmente en la península Ibérica por evidentes razones de índole socioeconómica. En ausencia de mega-herbívoros desbrozadores la matorralización parece muy difícil de controlar ya que el desbroce mecánico y el fuego son medidas costosas y poco efectivas a corto plazo que pueden tener consecuencias ecológicas muy negativas como la pérdida de suelo o la expansión de otras especies poco deseables (Calvo et al., 2002, 2005; Fernández Filgueira et al., 2013).

\subsection{Limitaciones de la información científica disponible para valorar la hipótesis plan- teada}

La mayoría de las cuestiones que se han tratado de resolver en la presente revisión constituyen aspectos fundamentales de debates científicos en curso, algunos de los cuales ya se prolongan más de un siglo. No tienen respuesta definitiva, ni siquiera algunas que parecen muy simples, como puede ser desentrañar las causas de la extinción de los grandes vertebrados terrestres a final del Pleistoceno. Parece que todavía estamos muy lejos de tener una respuesta clara para la mayoría de las preguntas que hemos planteado. En nuestra opinión, los principales obstáculos son: la sobrevaloración de los factores climáticos en detrimento de otros de gran importancia ecológica, como puede ser algún tipo de perturbación, y la extrema dificultad de reconstruir con 
precisión los ambientes naturales del pasado a partir de los análisis de los registros paleo-ecológicos.

A nuestro entender, la sobrevaloración del efecto del clima en la interpretación de la vegetación no es sólo un problema de la paleo-ecología sino que es mucho más general y también afecta a muchos estudios de ecología actual. Algunos de ellos, que tratan procesos actuales semejantes a los aquí considerados, también presentan resultados contradictorios e interpretaciones dispares. Seguidamente comentamos dos ejemplos que sugieren repensar los efectos del clima en los estudios de ecología vegetal:

El límite superior de los árboles en el Pirineo se considera por algunos autores muy determinado por la perturbación antrópica (Ameztegui et al., 2015) y por otros por las variaciones del clima (Camarero et al., 2015). Sin duda, este límite debe ser muy sensible a los cambios del clima al estar los árboles relativamente próximos a su límite de tolerancia fisiológica por frío y al riesgo de un periodo vegetativo excesivamente corto. Sin embargo, la perturbación también es muy importante ya que limita considerablemente la capacidad de estas plantas para tolerar el estrés (Grime, 1979), lo que debe rebajar, necesariamente, su límite altitudinal muy por debajo del máximo que existiría en condiciones de ausencia de perturbación (Fig. 1). En un pasado reciente este límite altitudinal estaría muy condicionado por las intensas perturbaciones del ganado y la importante presencia humana en el piso subalpino. Sin embargo, en las condiciones actuales de moderado régimen de perturbaciones, cabe esperar que tienda a ascender lentamente hasta la cota de equilibrio con el clima, el cual, a su vez, tiende a incrementar la temperatura, lo que debe elevar todavía más el límite altitudinal de los árboles.

Otro proceso que se observa en las últimas décadas en el Pirineo es el decaimiento de los abetales, cuyas causas no se han podido explicar satisfactoriamente (Oliva y Colinas, 2007). Se han formulado diversas hipótesis basadas en el parasitismo, la contaminación química, las sequías de las últimas décadas, algunos aspectos de explotación histórica (Camarero et al., 2002; Oliva y Colinas, 2007, 2010; Linares y Camarero, 2012; Sangüesa-Barreda et al., 2015; Camarero, 2017), el cambio de los usos del territorio (P. Montserrat, com. oral), etc. La abundancia y distribución del abeto está muy condicionada por su exigencia en fertilidad y humedad del suelo (Ruiz de la Torre, 1979) y, por este motivo, las fotografías de la figura 4 constituyen un buen ejemplo de la dificultad para explicar el estado de salud de los abetales en el paisaje actual. Los abetales que se supone más dependientes del clima, como son, por ejemplo, los de las cumbres de los montes Oroel y San Juan de la Peña (en la provincia de Huesca) con suelo somero y reducido aporte lateral de agua y nutrientes, se encuentran en perfectas condiciones y regeneran con vigor allí donde el viento o la nieve derriba algún ejemplar (Fig. 4a). Sin embargo, los abetales de ambientes muy frondosos y umbríos de pie de ladera que reciben importantes aportes de ésta, como los de las selvas de Villanúa y Castiello, cuyo estado era magnífico hace unas décadas, muestran ahora un conspicuo decaimiento (Fig. 4b). Observaciones como éstas parecen poco compatibles con las hipótesis que propugnan un déficit hídrico asociado al cambio climático, incluyendo el parasitismo de árboles debilitados por las sequías, pero se intuyen más fáciles de interpretar a partir de los cambios generales del uso del territorio que se experimentan desde la década de 1960. 

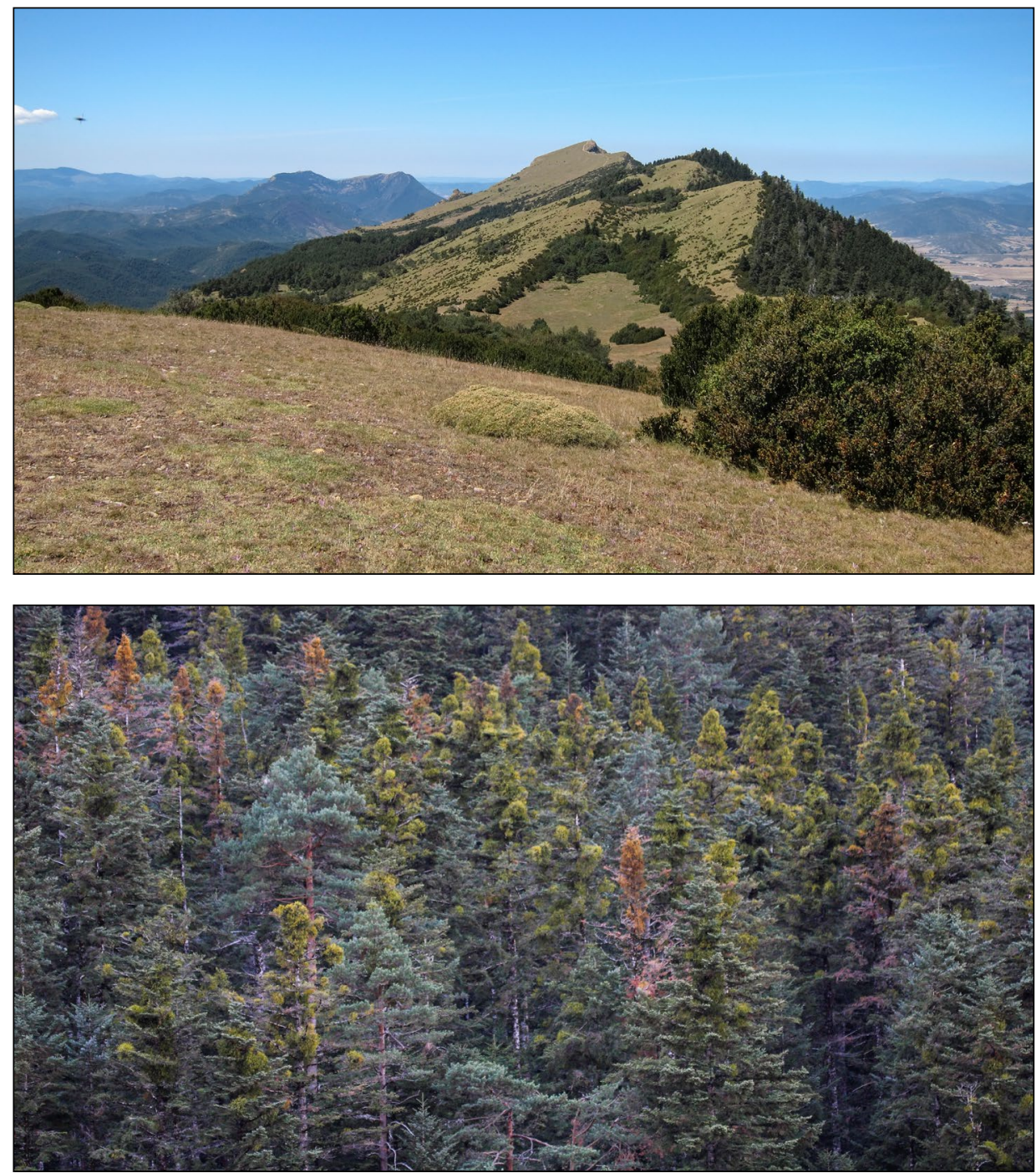

Figura 4. Abetales en distintas posiciones topográficas, distantes menos de $25 \mathrm{~km}$, en la Jacetania (provincia de Huesca). a). Cumbre del monte Oroel, 17 de agosto de 2017 (foto de Antonio Campo). b). Selva de Villanúa, 6 de noviembre de 2017 (foto de José Antonio Sesé Franco).

No parece que se puedan resolver problemas ecológicos como los planteados atendiendo solamente al clima y sin cuantificar cuidadosamente los demás factores ecológicos relevantes que los determinan.

Respecto a las dificultades para las reconstrucciones paleo-ecológicas, cabe señalar que estas interpretaciones de los ambientes pretéritos presentan muchos más problemas que los estudios ecológicos de los paisajes vegetales actuales. Pue- 
de ocurrir que el polen fósil produzca resultados distintos que los restos animales. Por ejemplo, en el sur de Europa durante el Pleistoceno temprano se reconstruyen paisajes de tipo sabana a partir de los restos animales, mientras que a partir del polen se interpreta que dominaba una vegetación forestal (Magri y Palombo, 2013). Esta discrepancia seguramente se debe a que se tiende a sobreestimar la presencia de las especies arbóreas en los registros de polen fósil (Bradshaw et al., 2003). También es reseñable la dificultad de estimar los regímenes de perturbación que actuaron en los lugares estudiados (Sugita, 2007a, 2007b; Soepboer y Lotter, 2009; Fyfe et al., 2013; Zanon et al., 2018). En los últimos años se han tratado de solventar algunas de estas limitaciones como, por ejemplo, la imposibilidad de medir la cobertura de las especies dominantes mediante el desarrollo de métodos que permiten cuantificar la cobertura de la vegetación en los registros paleo-palinológicos (LRA: landscape reconstruction algoritm, Sugita, 2007a, 2007b), aunque esta metodología todavía no se ha aplicado a la región mediterránea (Roberts et $a l ., 2018)$. Cabe suponer que en los próximos años veremos avances metodológicos muy notables pero las limitadas muestras de estudio seguirán dificultando el progreso. Si no somos capaces de entender bien las variaciones de las poblaciones que conforman el límite superior de los árboles y de especies como el abeto en el Pirineo, a pesar de tener acceso a material de estudio ilimitado en cualquier momento del año, ¿cómo podremos interpretar variaciones similares de los registros paleo-palinológicos en los que sólo disponemos de muestras escasas de unas pocas especies vegetales? Parece claro que todavía estamos muy lejos de poder disponer de reconstrucciones precisas de los principales procesos ecológicos y los paisajes vegetales que se produjeron en la península Ibérica durante los últimos 20.000 años.

Con todas las incertidumbres comentadas, esperamos que este artículo haga reconsiderar la importancia de las perturbaciones, además de los incendios, en la conformación y dinámica del paisaje vegetal. Concretamente, el efecto de los herbívoros grandes y medianos en la configuración del mosaico de pastos, matorral y bosque del paisaje vegetal natural. Mantener el equilibrio de dicho mosaico es esencial para un uso sostenible de los recursos de nuestras montañas y la conservación de las especies y los hábitats naturales.

\section{Agradecimientos}

Queremos agradecer a José María García Ruiz por habernos animado a redactar este trabajo. A José María Montserrat Martí, Sara Palacio y Javier Álvarez-Martínez por sus interesantes sugerencias para mejorar el manuscrito, a José Antonio Sesé Franco y Antonio Campo por aportarnos las fotografías del abetal de la Selva de Villanúa y de la cumbre del monte Oroel, respectivamente.

In memoriam de Pedro Montserrat Recoder que fue en nuestro país precursor de la interpretación y transmisión del papel que los herbívoros y la ganadería desempeñan en la formación y mantenimiento de unos "paisajes culturales" y en la conservación de la diversidad florística. 


\section{Bibliografía}

Aerts, R., Chapin III, F.S. 2000. The mineral nutrition of wild plants revisited: A re-evaluation of processes and patterns. Advances in Ecological Research 30, 1-67. https://doi.org/10.1016/ S0065-2504(08)60016-1.

Agustí, J., Antón, M. 2002. Mammoths, sabertooths, and hominids. 65 million years of mammalian evolution in Europe. Columbia University Press, New York, 313 pp.

Agustí, J., Antón, M. 2011. La gran migración. La evolución humana más allá de África. Crítica, Barcelona, $225 \mathrm{pp}$.

Alexander, K. 2005. Wood decay, insects, palaeoecology, and woodland conservation policy and practice: Breaking the halter. Antenna 29, 171-178.

Allen, J.R.M., Hickler, T., Singarayer, J.S., Sykes, M.T., Valdes, P.J., Huntley B. 2010. Last glacial vegetation of Northern Eurasia. Quaternary Science Reviews 29, 2604-2618. https:// doi.org/10.1016/j.quascirev.2010.05.31.

Álvarez-Lao, D.J., García, N. 2010. Chronological distribution of Pleistocene cold-adapted large mammal faunas in the Iberian Peninsula. Quaternary International 212, 120-128. https://doi. org/10.1111/j.quaint.2009.02.029.

Amenztegui, A., Coll, L., Brotons, L., Ninot, J.M. 2015. Land-use legacies rather than climate change are driving the recent upward shift of the mountain tree line in the Pyrenees. Global Ecology and Biogeography 25, 263-273. https://doi.org/10.1111/geb.12407.

Aranbarri, J., González-Sampériz, P., Valero-Garcés, B., Moreno, A., Gil-Romera, G., Sevilla-Callejo, M., García-Prieto, E., Di Rita, F., Mata, M.P., Morellón, M., Magri, D., Rodríguez-Lázaro, J., Carrión, J.S. 2014. Rapid climatic changes and resilient vegetation during the Lateglacial and Holocene in a continental region of southwestern Europe. Global and Planetary Change 114, 50-65. https://doi.org/10.1016/j. gloplacha.2014.01.003.

Aseginolaza Iparragirre, C., Gómez García, D., Lizaur Sukia, X., Montserrat Martí,, G., Morante Serrano G., et al. 1984. Catálogo florístico de Álava, Vizcaya y Guipúzcoa. Gobierno Vasco. Viceconsejería de Medio Ambiente, Vitoria-Gasteiz, 1149 pp.

Asner, G.P., Levick, S.R. 2012 Landscape-scale effects of herbivores on treefall in African savannas. Ecology Letters 15, 1211-1217. https://doi.org/10.1111/j.1461-0248.2012.01842.x.

Asner, G.P., Vaughn, N., Smit, I.P.J., Levick, S.L. 2016. Ecosystem-scale effects of megafauna in African savannas. Ecography 39, 240-252. https://doi.org/10.1111/ecog.01640.

Atwell, L., Kovarovic, K., Kendal, J.R. 2015. Fire in the Plio-Pleistocene: the functions of hominin fire use, and the mechanistic, developmental and evolutionary consequences. Journal of Anthropological Sciences 93, 1-20.

Bakker, E.S., Gill, J.L., Johnson, C.N., Vera, F.W.M., Sandom, C.J., Asner, G.P., Svenning, J.C. 2016. Combining paleo-data and modern exclosure experiments to asses the impact of megafauna extinctions on woody vegetation. PNAS 11 (4)3,847-855. https://doi.org/10.1073/ pnas. 1502545112 .

Barnosky, A.D., Lindsey, E.L., Villavicencio, N.A., Bostelmann, E., Hadly, E.A., et al. 2016. Variable impact of late-Quaternary magafaunal extinction in causing ecological state shifts in North and South America. PNAS 113 (4), 856-861. https://doi.org/10.1073/pnas.1505295112.

Bartlett, L.J., Williams, D.R., Prescott, G.W., Balmford, A., Green, R.E., Eriksson, A., Valdes, P.J.,Singarayer, J.S., Manica, A. 2016. Robustness despite uncertainty: regional climate data reveal the dominant role of humans in explaining global extinctions of Late Quaternary megafauna. Ecography 39, 152-161. https://doi.org/10.1111/ecog.01566. 
Bartolomé, J., Franch, J., Plaixats, J., Seligman, N.G. 2000. Grazing alone is not enough to maintain landscape diversity in the Montseny Biosphere Reserve. Agriculture, Ecosystems and Environment 77, 267-273. https://doi.org/10.1016/S0167-8809(99)00086-9.

Benito Garzón, M., Sánchez de Dios, R., Sáinz Ollero, H. 2007. Predictive modelling of tree species distributions on the Iberian Peninsula during the Last Glacial Maximum and MidHolocene. Ecography 30, 120-134. https://doi.org/10.1111/j.0906-7590.2007.04813.x

Berglund, B.E., Birks, H.J.B., Ralska-Jasiewiczowa, M., Wright, H.E., Eds. 1996. Palaeoecological events during the last 15000 years: regional synthesis of palaeoecological studies in lakes and mires in Europe. John Wiley \& Sons Ltd., Chichester.

Binney, H., Edwards, M., Macias-Fauria, M., Lozhkin, A., Anderson, P., Kaplan, J.O., Andreev, A., Bezrukova, E., Blyakharchuk, T., Jankovska, V., Khazina, I., Krivonogov, S., Kremenetski, K., Nield, J., Novenko, E., Ryabogina, N., Solovieva, N., Willis, K., Zernitskaya, V. 2017. Vegetation of Eurasia from the last glacial maximum to present: Key biogeographic patterns. Quaternary Science Reviews 157, 80-97. https://doi.org/10.1016/j.quascirev.2016.11.022.

Birks, H.J.B. 2005. Mind the gap: how open were European primeval forests? Trends in Ecology and Evolution 20, 154-156. https://doi.org/10.1016/j.tree.2005.02.001.

Blanca, G., Cabezudo, B., Cueto, M., Salazar, C., Morales Torres, C., Eds. 2011. Flora vascular de Andalucía Oriental: Universidades de Almería, Granada, Jaén y Málaga. 1751 pp., 2 ed.

Bocherens, H., Hofman-Kaminska, E., Drucker, D.G., Schmölcke, U., Kowalczyk, R. 2015. European bison as a refugee species? Evidence from isotopic data on early Holocene bison and other large herbivores in Northern Europe. PLOS ONE https://doi.org/10.1371/journal.pone.0115090.

Bond, W. 2010. Consumer control by megafauna and fire. En: J. Terborgh, J.A. Estes (Eds.), Trophic cascades: predators, prey and the changing dynamics of nature. Island Press, Washington, pp. 275-286.

Bond, W., Keeley, J.E. 2005. Fire as a global "herbivore": the ecology and evolution of flammable ecosystems. Trends in Ecology and Evolution 20, 387-394. https://doi.org/10.1016/j. tree.2005.04.025.

Bond, W., Woodward, F.I., Midgley, G.F. 2005. The global distribution of ecosystems in a world without fire. New Phytologist 165, 525-538. https://doi.org/10.1111/j.14698137.2004.01252.x.

Bradshaw, R.H.W., Hannon, G.E., Lister, A.M. 2003. A long-term perspective on ungulatevegetation interactions. Forest Ecology and Management 181, 267-280. https://doi. org/10.1016/S0378-1127(03)00138-5.

Bredenkamp,G.J.,Spada,F.,Kazmierczak,E.2002.On the origin of northern and southernhemisphere grasslands. Plant Ecology 163, 209-229. https://doi.org/10.1023/A:1020957807971.

Britton, K., Grimes, V., Niven, L., Steele, T.E., McPeherron, S., Soressi, M., Kelly, T.E., Jaubert, J., Hublin, J.J., Richards, M.P. 2011. Strontium isotope evidence for migration in late Pleistocene Rangifer: Implications for Neanderthal hunting strategies at the Middle Palaeolithic site of Jonzac, France. Journal of Human Evolution 61, 176-185. https://doi. org/10.1016/j.hevol.2011.03.004.

Calvo, L., Tárrega, R., De Luis, E. 2002. Secondary succession after perturbations in a shrubland community. Acta Oecologica 23, 393-404. https://doi.org/10.1016/S1146-609X(02)01164-5.

Calvo, L., Tárrega, R., De Luis, E., Valbuena, L., Marcos, E. 2005. Recovery after experimental cutting and burning in three shrub communities with different dominant species. Plant Ecology 180: 175-185. https://doi.org/10.1007/s11258-005-0200-z.

Camarero, J.J. 2017. The multiple factors explaining decline in mountain forests: historical logging and warming-related drought stress is causing silver-fir dieback in the Aragón Pyrenees. En: J. Catalán, J.M. Ninot, M.M. Aniz (Eds.), High mountain conservation in a changing world. 
Gewerbestrasse: Springer, Gewerbestrasse, pp. 131-156. https://doi.org/10.1007/978-3-31955982-7_6.

Camarero J.J., García-Ruiz J.M., Sangüesa Barreda G., Galván J.D., Alla A.Q., Sanjuán, Y., Beguería, S., Gutiérrez, E. 2015. Recent and intense dynamics in a formerly static Pyrenean treeline. Arctic, Antarctic and Alpine Research 47, 773-83. http://doi.org/10.1657/ AAAR0015-001.

Camarero, J.J., Padró, A., Martín, E., Gil-Pelegrín, E. 2002. Aproximación dendroecológica al decaimiento del abeto (Abies alba Mill.) en el Pirineo Aragonés. Montes 70, 26-33.

Carey, J. 2016. Rewilding. Proceedings of the National Academy of Sciences of the United States of America 113, 806-808. https://doi.org/10.1073/pnas.1522151112.

Carotenuto, F., Di Febbraro, M., Melchionna, M., Castiglione, S., Saggese, F., Serio, C., Mondanaro, A., Passaro, F., Loy, A., Raia, P. 2016. The influence of climate on species distribution over time and space during the late Quaternary. Quaternary Science Reviews 149, 188-199. https://doi.org/10.1016/j.quascirev.2016.07.036.

Carrión, J.S. 2002. Patterns and processes of Late Quaternary environmental change in a mountain region of southwestern Europe. Quaternary Science Reviews 21, 2047-2066. https://doi. org/10.1016/S0277-3791(02)00010-0.

Carrión, J.S., Fernández, S., González-Sampériz, P., Gil-Romera, G., Badal, E., Carrión-Marco, Y., López-Merino, L., López-Sáez, J.A., Fierro, E., Burjachs, F. 2010 a. Expected trends and surprises in the Lateglacial and Holocene vegetation history of the Iberian Peninsula and Balearic Islands. Review of Paleobotany and Palynology 162, 458-475. https://doi. org/10.1016/j.revpalbo.2009.12.007.

Carrión, J.S., Fernández, S., Jiménez- Moreno, G., Fauquette, S., Gil-Romera, G., et al. 2010 b. The historical origins of aridity and vegetation degradation in southeastern Spain. Journal of Arid Environments 74,731-736. https://doi.org/10.1016/j.jaridenv.2008.11.014.

Carrión J.S., Fuentes N., González-Sampériz P., Sánchez Quirante L., Finlayson J.C., Fernández, S., Andrade, A. 2007. Holocene environmental change in a montane region of southern Europe with a long history of human settlement. Quaternary Science Reviews 26, 1455-1475. https://doi.org/10.1016/j.quascirev.2007.03.013.

Castroviejo, S. 1986-2015. Flora Iberica. Real Jardín Botánico, Madrid.

Churchill, S.E., Rhodes, J.A. 2009. The evolution of the human capacity for "killing at a distance": The human fossil evidence for the evolution of projectile weaponry. En: J.J. Hublin, M.P. Richards (Eds.), The evolution of hominin diets: integrating approaches to the study of Palaeolithic subsistence. Springer, pp. 201-210. https://doi.org/10.1007/978-1-4020-9699-0_15.

Collantes Gutiérrez, F. 2003. La ganadería de montaña en España, 1865-2000: Historia de una ventaja comparativa anulada. Historia agraria 31, 141-167.

Collins, P.M., Davis, B.A.S., Kaplan, J.O. 2012. The mid-Holocene vegetation of the Mediterranean region and southern Europe, and comparison with the present day. Journal of Biogeography 39, 1848-1861. https://doi.org/10.1111/j.1365-2699.2012.02738.x.

Conant, R.T., Easter, M., Paustian, K., Swan, A., Williams S. 2007. Impacts of periodic tillage on soil C stocks: A synthesis. Soil and Tillage Research 95, 1-10. https://doi.org/10.1016/j. still.2006.12.006.

Coope, G.R. 2000. Middle Devensian (Weichselian) coleopteran assemblages from Earith, Cambridgeshire (UK) and their bearing on the interpretation of "Full glacial" floras and faunas. Journal of Quaternary Science 15, 779-788. https://doi.org/10.1002/10991417(200012)15:8<779::AID-JQS565>3.0CO;2-H.

Cortés Sánchez, M., Jiménez Espejo, F.J., Simón Vallejo, M.D., Gibaja Bao, J.F., Carvalho, A.F., Martínez-Ruiz, F., Rodrigo Gámiz, M., Floires, J.A., Paytan, A., López-Sáez, J.A., PeñaChocarro, L., Xarrión, J.S., Morales Muñiz, A., Roselló Izquierdo, E., Riquelme Cantal, 
J.A., Dean, R.M., Salgueiro, E., Martínez Sánchez, R.M., Bicho, N.F. 2012. The MesolithicNeolithic transition in southern Iberia. Quaternary Research 77, 221-234. https://doi. org/10.1016/j.yqres.2011.12.003.

Cunill, R., Soriano, J.M., Bal, M.C., Pèlach, A., Pérez-Obiol, R. 2012. Holocene treeline changes on the south slope of the Pyrenees: a pedoanthracological analysis. Vegetation History and Archaeobotany 21, 373-384. https://doi.org/10.1007/s00334-011-0342-y.

Cunill, R., Soriano, J.M., Bal, M.C., Pèlachs, A., Rodríguez, J.M., Pérez-Obiol, R. 2013. Holocene high-altitude vegetation dynamics in the Pyrenees: A pedoanthracology contribution to an interdisciplinary approach. Quaternary International 289, 60-70. https://doi.org/10.1016/j. quaint.2012.04.041.

Demment, M.W., Van Soest, P.J. 1985. A nutritional explanation for body-size patterns of rumiant and nonrumiant herbivores. The American Naturalist 125, 641-672. https://doi. org/10.1086/284369.

Dering, M., Latalowa, M., Boratynska, K., Kosinski, P., Boratynski, A. 2016. Could clonality contribute to the northern survival of grey alder [Alnus incana (L.) Moench] during the Last Glacial Maximum? Acta Societatis Botanicorum Poloniae 86 (3523). https://doi.org/10.5586/asbp.3523.

Dias, R., Detry, C., Bicho, N. 2016. Changes in the exploitation dynamics of small terrestrial vertebrates and fish during the Pleistocene-Holocene transition in the SW Iberian Peninsula: A reiew. The Holocene 26, 964-984. https://doi.org/10.1177/0959683615622547.

Dinnin, M.H., Sadler, J.P. 1999. 10,000 years of change: the Holocene entomofauna of the British Isles. En: K.J. Edwards, J.P. Sadler (Eds.), Holocene environments of prehistoric Britain, Quaternary Proceedings 7. John Wiley, Chichester, pp. 545-562. https://doi.org/10.1002/ (SICI) 1099-1417(199910) 14:6<545::AID-JQS489>3.0.CO;2-X.

Doughty, C.E., Malhi, Y. 2013. The legacy of the Pleistocene megafauna extinctions on nutrient availability in Amazonia. Nature Geoscience 6, 761-764. https://doi.org/10.1038/ngeo.1895.

Doughty, C.E., Wolf, A., Field, C.B. 2010. Biophysical feedbacks between the Pleistocene megafauna extiction and climate: The first human-induced global warming? Geophysical Research Letters 37. https://doi.org/10.1029/2010GL043985.

Drucker, D., Bocherens, H., Bridaut, A., Billiou, D. 2003. Carbon and nitrogen isotopic composition of red deer (Cervus elaphus) collagen as a tool for tracking paleoenvironmental change during the late-Glacial and Early Holocene in northern Jura (France). Palaeography, Palaeoclimatology, Palaeoecology 195, 375-388. https://doi.org/10.1016/S00310182(03)00366-3.

Drucker, D.G., Bridault, A., Hobson, K.A., Szuma, E., Bocherens, H. 2008. Can carbon-13 in large herbivores reflect the canopy effect in temperate and borela ecosystems? Evidence from modern and ancient ungulates. Palaeography, Palaeoclimatology, Palaeoecology 266, 69-82. https://doi.org/10.1016/j.palaeo.2008.03.020.

Ellemberg, H. 1988. Vegetation ecology of central Europe. Cambridge University Press, Cambridge, $731 \mathrm{pp}$.

Fernández Filgueira, C., Vega Hidalgo, J. A., Fontube Lliteras, T. 2013. Cambios en la cobertura vegetal después de tratamientos preventivos de incendios forestales en dos áreas de matorral del Norte de España. $6^{\circ}$ Congreso forestal español. Sociedad de Ciencias Forestales. VitoriaGasteiz ISBN: 978-84-937964-9-5.

Fernández-López de Pablo, J., Gabriel, S. 2016. El Collado shell midden and the exploitation patterns of littoral resources during the Mesolithic in the Eastern Iberian Peninsula. Quaternary International 407, 106-117. https://doi.org/10.1016/j.quaint.2015.11.100.

Firbas, F. 1949. Sät und nacheiszeitliche Waldegeschichte Mitteleuropas nördlich der Alpen. Gustav Fischer. 
Flodin, N.W. 1999. Nutritional influences in the geographic dispersal of Pleistocene man. Ecology of Food and Nutrition 38, 71-99. https://doi.org/10.1080/03670244.1999.9991571.

Fyfe, R.M., Twiddle, C., Sugita, S., Gaillard, M.J., Barratt, P., Caseldine, C.J., Dodson, J., Edwards, K.J., Farrell, M., Froyd, C., Grant, M.J., Huckerby, E., Innes, J.B., Shaw, H., Waller, M. 2013. The Holocene vegetation cover of Britain and Ireland: overcoming problems of scale and discerning patterns of openness. Quaternary Science Reviews 73, 132-48. https://doi. org/10.1016/j.quascirev.2013.05.014.

García-González, R. 2008. La utilización de los pastos por los grandes herbívoros: principios básicos y casos de estudio. En: F. Fillat, R. García-González, D. Gómez, R. Reiné, Pastos del Pirineo, Consejo Superior de Investigaciones Científicas y Diputación de Huesca, pp. 205-227.

García-Ruiz J.M., Sanjuán Y., Gil-Romera G., González-Sampériz P., Beguería S., Arnáez, J., Coba-Pérez, P., Gómez-Villar, A., Álvarez-Martínez, J., Lana-Renault, N., Pérez-Cardiel, E., López de Calle, C. 2016. Mid and late Holocene forest fires and deforestation in the subalpine belt of the Iberian Range, northern Spain. Journal of Mountain Science 13, 176072. http://doi.org/10.1007/s11629-015-3763-8.

Giesecke, T. 2016. Did thermophilous trees spread into central Europe during the Late Glacial? New Phytologist 212, 15-18. https://doi.org/10.111/nph.14149.

Gil-Romera, G., Carrión, J.S., Pausas, J.G., Sevilla-Callejo, M., Lamb, H.F., Fernández, S., Burjachs, F. 2010. Holocene fire activity and vegetation response in South-Eastern Iberia. Quaternary Science Reviews 29, 1082-1092.https://doi.org/10.1016/j.quascirev.2010.01.006.

Gil-Romera, G., González-Sampériz, P., Lasheras-Álvarez, L., Sevilla-Callejo, M., Moreno, A., Valero-Garcés, B., López-Merino, L., Carrión, J.S., Pérez Sanz, A., Aranbarri, J., GarcíaPrieto Fronce, E. 2014. Biomass-modulated fire dynamics during the Last Gacial-Interglacial Transition at the Central Pyrenees (Spain). Palaeography, Palaeoclimatology, Palaeoecology 402, 113-124. https://doi.org/10.1016/j.palaeo.2014.03.015.

Gill, J.L. 2014. Ecological impacts of the late Quaternary magaherbivore extinctions. New Phytologist 201, 1163-1169. https://doi.org/10.1111/nph.12576.

Gill, J.L., McLauchlan, K.K., Skibbe, A.M., Goring, S.J., Williams, J.W. 2013. Linking abundances of the dung fungud Sporormiella to the density of Bison: Implications for assesing grazing by megaherbivores in paleorecords. Journal of Ecology 101, 1125-1136. https://doi.org/10.1111/1365-2745.12130.

Gill, J.L., Williams, J.W., Jackson, S.T., Donnelly, J.P., Schellinger, G.C. 2012. Climatic and megaherbivory controls on late-glacial vegetation dynamics: A new, high-resolution, multiproxy record from Silver Lake, Ohio Quaternary Science Reviews 34, 68-80. https://doi. org/10.1016/j.quascirev.2011.12.008.

Gill, J.L., Williams, J.W., Jackson, S.T., Lininger, K.B., Robinson, G.S. 2009. Pleistocene megafaunal collapse, novel plant communities, and enhanced fire regimes in North America. Science 326 (5956), 1100-1103. https://doi.org/10.1126/science.1179504.

Gómez García, D., Mateo, G., Mercadal, N., Montserrat, P., Sesé, J.A. 2005. Atlas digital de la Flora de Aragón. http://floragon.ipe.csic.es

Gómez García, D., Aguirre, A.J., Jiménez, R., Manrique, C. en revisión. Shrub encroachment in Mediterranean mountain grasslands: rate and consequences on plant diversity and forage availability. Rangeland Ecology and Management (en prensa).

Gómez Gutiérrez, J.M., González Bartolomé, R., Fernández Santos, B., Galindo Villardón, P. 1988. Regeneración post-fuego del piornal serrano. Formaciones de Cytisus balansae (Boiss.) Ball. Anuario del Centro de Edafología y Biología Aplicada del CSIC 13, 261-277.

González-Fortes, G., Jones, E.R., Lightfoot, E., Bonsall, C., Lazar, C., Grandal d'Anglade, A., Garralda, M.D., Drak, L., Simalcsik, A., Boroneant, A., Vidal Romaní, J.R., Vaqueiro 
Rodríguez, M., Arias, P., Pinhasi, R., Manica, A., Hofreiter, M. 2017. Paleogenomic evidence for multi-generational mixing between neolithic farmers and mesolithic hunter-gatherers in the Lower Danube Basin. Current Biology 27, 1801-1810. https://doi.org/10.1016/j. cub.2017.05.023.

González-Guerrero, O., Cruañas Cruells, G., Ninyerola Casals, M. 2017. Análisis de la dinámica espacio-temporal (1948-2003) del límite altitudinal del bosque (tree line) en Andorra mediante fotografía aérea. GeoFocus 19, 3-25. https://doi.org/10.21138/GF.458.

González-Sampériz, P., Aranbarri, J., Pérez-Sanz, A., Gil-Romera, G., Moreno, A., Leunda, M., Sevilla-Callejo, M., Corella, J.P., Morellón, M., Oliva, B., Valero-Garcés, B. 2017. Environmental and climate change in the southern Central Pyrenees since the Last Glacial Maximum: A review from the lake records. Catena 149, 668-688. https://doi.org/10.1016/j. catena.2016.07.041.

González-Sampériz, P., Leroy, S., Carrión, J.S., Fernández, S., García-Antón, M., Gil-García, M.J., Uzquiano, P., Valero-Garcés, B., Figueiral, I. 2010. Steppes, savannahs, forests and phytodiversity reservoirs during the Pleistocene in the Iberian Peninsula. Review of Paleobotany and Palynology 162, 427-457. https://doi.org/10.1016/j.revpalbo.2010.03.009.

González-Sampériz, P., Valero-Garcés, B., Moreno, A., Morellón, M., Navas, A., Machín, J., Delgado-Huertas, A. 2008. Vegetation changes and hydrological fluctuations in the Central Ebro Basin (NE Spain) since the Late Glacial period: Saline lake records. Palaeography, Palaeoclimatology, Palaeoecology 259, 157-181. https://doi.org/10.1016/j. palaeo.2007.10.005.

Graham, R.W., Lundelius, E.L. 1984. Coevolutionary disequilibrium and Pleistocene extinctions. En: P.S. Martin, R.G. Klein (Eds.), Quaternary extinctions: A prehistoric revolution. University of Arizona Press, Tucson, pp. 223-249.

Grime, J.P. 1979. Plant strategies and vegetation processes. John Wiley, Chichester, 222 pp.

Grime, J.P., Hodgson, J.G., Hunt, R. 2007. Comparative plant ecology. A functional approach to common British species. Castlepoint Press Colvend, 748 pp. 2 ed.

Guimaraes, P.R., Galetti, M., Jordano, P. 2008. Seed dispersal anachronisms: rethinking the fruits extinct megafauna ate. PLOS ONE 3,(3), e17745. https://doi.org/10.1371/journal. pone.0001745.

Guthrie, R.D. 1984. Mosaics, allelochemics, and nutients: an ecological theory of late Pleistocene megafaunal extinctions. En: P.S. Martin, R.G. Klein (Eds.), Quaternary extinctions: A prehistoric revolution. University of Arizona Press, Tucson, pp. 259-298.

Guthrie, R.D. 2001. Origin and causes of the mammoth steppe: a story of cloud cover, woolly mammoth tooth pits, buckles, and inside-out Beringia. Quaternary Science Reviews 20, 549574. https://doi.org/10.1016/S0277-3791(00)00099-8.

Hejcman, M., Hejcmanová, P., Pavlu, V., Benes, J. 2013. Origin and history of grasslands in Central Europe- a review. Grass and Forage Science 68, 345-363. https://doi.org/10.1111/ gfs. 12066 .

Herrero, J., García-González, R., García-Serrano, A. 1994. Altitudinal distribution of alpine marmot (Marmota marmota) in the Pyrenees, Spain/France. Arctic and Alpine Research 26, 328-331. https://doi.org/10.2307/1551793.

Hervella, M., Svensson, E.M., Alberdi, A., Günthe,r T., Izaguirre, N., Munters, A.R., Alonso, S., Ioana, M., Ridiche, F., Soficaru, A., Jacobsson, M., Netea, M.G., de-la-Rua, C. 2016. The mitogenome of a 35,000-year-old Homo sapiens from Europe supports a Paleolithic back-migration to Africa. Nature Comunications / Scientific Reports 6, 25501. https://doi. $\operatorname{org} / 10.1038 /$ srep25501. 
Hofman-Kaminska, E., Bocherens, H., Borowik, T., Drucker, D.G., Kowalczyk, R. 2018. Stable isotope signatures of large herbivore foraging habitats across Europe. PLOS ONE. https:// doi.org/10.1371/journal.pone.0190723.

Hofmann, R.R. 1989. Evolutionary steps of ecophysiological adaptation and diversification of ruminants: a comparative view of their digestive system. Oecologia 78, 443-457. https://doi. org/10.1007/BF00378733.

Hoppe, K.A., Koch, P.L. 2007. Reconstructing the migration patterns of late Pleistocene mammals from northern Florida, USA. Quaternary Research 68, 347-352. https://doi.org/10.1016/j. yqres.2007.08.001.

Huntley, B., Allen, J.R.M., Collingham, Y.C., Hickler, T., Lister, A.M., Singarayer, J., Stuart, A.J., Sykes, M.T., Valdes, P.J. 2013. Millenial climatic fluctuations are key to the structure of Last Glacial ecosystems. PLOS ONE 8 (4), e61963. https://doi.org/10.1371/journal. pone.0061963.

Huntley, B., Birks, H.J.B. 1983. An atlas of past and present maps for Europe: 0-13.000 years ago. Cambridge University Press, Cambridge.

Innes, J.B., Blackford, J.J., Rowley-Conwy, P.A. 2013. Late Mesolithic and early Neolithic forest disturbance: a high resolution palaeoecological test of human impact hypotheses. Quaternary Science Reviews 77, 80-100. https://doi.org/10.1016/j.quascirev.3013.07.012.

Iversen, J. 1960. Problems of the early post-glacial forest development in Denmark. Danm. Geol. Unders. Ser. IV 4, 1-32.

Johnson, C.N. 2009. Ecological consequences of late Quaternary extinctions of megafauna. Proceedings of the Royal Society, London B276, 2509-2519. https://doi.org/10.1098/ rspb.2008.1921.

Johnson, C.N., Rule, S., Haberle, S.G., Kershaw, A.P., McKenzie, G.M., Brook, B.W. 2016. Geographic variation in the ecological effects of extinction of Australia's Pleistocene megafauna. Ecography 39, 109-116. https://doi.org/10.1111/ecog.01612.

Jouzel, J., Masson-Delmotte, V., Cattani, O., Dreyfus, G., Falourd, S., Hoffmann, G., Minster, B., Nouet, J., Barnola, J.M., Chappellaz, J., Fischer, H., Gallet, J.C., Johnsen, S., Leuenberger, M., Loulergue, L., Luethi, D., Oerter, H., Parrenin, F., Raisbeck, G., Raynaud, D., Schilt, A., Schwander, J., Selmo, E., Souchez, R., Spahni, R., Stauffer, B., Steffensen, J.P., Stenni, B., Stocker, T.F., Tison, J.L., Werner, M., Wolff, E.W. 2007. Orbital and millennial Antarctic climate variability over the past 800.000 years. Science $317,793-796$. https://doi.org/10.1126/ science. 1141038.

Julien, M.A., Bocherens, H., Burke, A., Drucker, D.G., Patou-Mathis, M., Krotova, O., Péan, S. 2012. Were European steppe bison migratory? 18O, 13C and $\mathrm{Sr}$ intra-tooth isotopic variations applied to palaeoethological reconstruction. Quaternary International 271, 106119. https://doi.org/10.1016/j.quaint.2012.06.011.

Juricková, L., Horácková, J., Lozek, V. 2014. Direct evidence of central European forest refugia during the last glacial period based on mollusc fossils. Quaternary Research 82, 222-228. https://doi.org/10.1016/j.yqres.2014.01.015.

Kaal, J., Carrión Marco, Y., Asouti, E., Martín Seijo, M., Martínez Cortizas, A., Costa Casáis, M., Criado Boado, F. 2011. Long-term deforestation in NW Spain: linking the Holocene fire history to vegetation change and human activities. Quaternary Science Reviews 30, 161-175. https://doi.org/10.1016/j.quascirev.2010.10.006.

Kaplan, J.O., Pfeiffer, M., Kolen, J.C.A., Davis, B.A.S. 2016. Large scale anthropogenic reduction of forest cover in Last Glacial Maximum Europe. PLOS ONE. https://doi.org/10.1371/ journal.pone.0166726.

Keen, D.H., Bateman, M.D., Coope, G.R., Field, H., Langford, H.E., Merry, J.S., Mighall, T.S. 1999. Sedimentology, palaeoecology and geochronology of Last Interglacial deposits from 
Deeping St James, Lincolnshire, England. Journal of Quaternary Science 14, 411-436. https://doi.org/1002/(SICI)1099-1417(199908)14:5<411::AID-JQS447>3.0CO;2-M.

Koch, P.L., Barnosky, A.D. 2006. Late Quaternary extinctions: state of the debate. Annual Reviews of Ecology, Evolution and Systematics 37, 215-225. https://doi.org/10.1146/annurev. ecolsys.34.011802.132415.

Kolstrup, E. 1990. The puzzle of Weichselian vegetation types poor in trees. Geologie en Mijnbouw 69, 253-262.

Kukla, G. 2005. Saalian supercycle, Mindel/Riss interglacial and Milankovitch's dating. Quaternary Science Reviews 24, 1573-1583. https://doi.org/10.1016/j.quascirev.2004.08.023.

Kunes, P., Odgaard, B.V., Gaillard, M.J. 2011. Soil phosphorous as a control of productivity and openess in temperate interglacial forest ecosystems. Journal of Biogeography 38, 2150-2164. https://doi.org/10.1111/j.1365-2699.2011.02557.x.

Kunes, P., Svobodová-Svitavská, H., Kolár, J., Hajnalová, M., Abraham, V., Macek, M., Tkác, P., Szabó, P. 2015. The origin of grasslands in the temperate forest zone of east-central Europe: long-term legacy of climate and human impact. Quaternary Science Reviews 116, 15-27. https://doi.org/10.1016/j.quascirev.2015.03.014.

Lawson, I.T., Tzedakis, P.C., Roucoux, K.H., Galanidou, N. 2013. The anthropogenic influence on wildfire regimes: charcoal records from the Holocene and last interglacial at Ioannina, Grece. Journal of Biogeography 40, 2324-2334. https://doi.org/10.1111/jbi.12164.

Lehndorff, E., Wolf, M., Litt, T., Brauer, A., Amelung, W. 2015. 15,000 years of black carbon deposition- A post glacial fire record from maar lake sediments (Germany). Quaternary Science Reviews 110, 15-22. https://doi.org/10.1016/j.quascirev.2014.12.014.

Linares, J.C., Camarero, J.J. 2012. Growth patterns and sensitivity to climate predict silver fir decline in the Spanish Pyrenees. European Journal of Forest Research 131, 1001-1012. https://doi.org/10.1007/s10342-011-0572-7.

López Sáez, J.A., Abel-Schaad, D., Pérez-Díaz, S., Blanco-González, A., Alba-Sánchez, F., Dorado, M., Ruiz-Zapata, B., Gil-García, M.J., Gómez-González, C., Franco-Múgica, F. 2014. Vegetation history, climate and human impact in the Spanish Central System over the last 9000 years. Quaternary International 353, 98-122. https://doi.org/10.1016/j.quaint.2013.06.034.

López Sáez, J.A., López Merino, L., Alba Sánchez, F., Pérz Díaz, S. 2009. Contribución paleoambiental al estudio de la trashumancia en el sector abulense de la Sierra de Gredos. Hispania 69, 9-38. https://doi.org/10.3989/hispania.2009.v69.i231.97.

MacDonald, G.M., Beilman, D.W., Kuzmin, Y.V., Orlova, L.A., Kremenetski, K.V., Shapiro, B., Wayne, R.K., Van Valkenburgh, B. 2012. Pattern of extinction of the woolly mammoth in Beringia. Nature Communications 3(893), 1-8. https://doi.org/10.1038/ncomms1881.

Magri, D., Palombo, M.R. 2013. Early to Moddle Pleistocene dynamics of plant and mamal communities in South West Europe. Quaternary International 288, 63-72. https://doi. org/10.1016/j.quaint.2012.02.028.

Malhi, Y., Doughty, C.E., Galetti, M., Smith, F.A., Svenning, J.C., Terborgh, J.W. 2016. Megafauna and ecosystem function from the Pleistocene to the Anthropocene. PNAS 113, 838-846. https://doi.org/10.1073/pnas.1502540113.

Mann, D.H., Groves, P., Kunz, M.L., Reanier, R.E., Gaglioti, B.V. 2013. Ice-age megafauna in artic Alaska: Extinction, invasion, survival. Quaternary Science Reviews 70, 91-108. https:// doi.org/10.1016/j.quascirev.2013.03.015.

Marín-Arroyo, A.B. 2013. Human response to Holocene warming on the Cantabrian Coast (northern Spain): an unexpected outcome. Quaternary Science Reviews 81, 1-11. https://doi. org/10.1016/j.quascirev.2013.09.006.

Markova, A.K., Puzachenko, A.Y., van Kolfschoten, T., van der Plicht, J., Ponomarev, D.V. 2013. New data on changes in the European distribution of mammoth and the woolly rhinoceros 
during the second half of the Pleistocene and the early Holocene. Quaternary International 29, 4-14. https://doi.org/10.1016/j.quaint.2012.11.033.

Marlon, J.R., Bartlein, P.J., Daniau, A.L., Harrison, S.P, Maezumi, S.Y., Power, M.J., Tinner, W., Vannière, B. 2013. Global biomass burning: a synthesis and review of Holocene paleofire records and their controls. Quaternary Science Reviews 65, 5-25. https://doi.org/10.1016/j. quascirev.2012.11.029.

Martin, P.S. 1967. Prehistoric overkill. En: P.S. Martin (Ed.), Pleitocene extinctions the search for a cause. Yale University Press, New Heaven, pp. 354-403.

Martin, P.S., Steadman, D.W. 1999. Prehistoric Extinctions on Islands and Continents. En: R.D.E. MacPhee (Eds.) Extinctions in Near Time. Advances in Vertebrate Paleobiology, vol 2. Springer, Boston. https://doi.org/10.1007/978-1-4757-5202-1_2.

Mason, S.L.R. 2000. Fire and Mesolithic subsistence-managing oaks for acorns in northwest Europe? Palaeography, Palaeoclimatology, Palaeoecology 164, 139-150. https://doi. org/10.1016/S0031-0182(00)00181-4.

Mitchell, F.J. 2005. How Open were European primeval forests? Hypothesis testing using palaeoecological data. Journal of Ecology 93, 168-177. https://doi.org/10.1111/j.13652745.2004.00964.x.

Mix, A.C., Bard, E., Schneider, R. 2001. Environmental processes of the ice age: land, oceans, glaciers (EPILOG). Quaternary Science Reviews 20, 627-657. https://doi.org/10.1016/ S0277-3791(00)00145-1.

Montserrat Martí, G. 1986. Datos para el estudio de la alianza Aphyllanthion Br.-Bl. en el Prepirineo aragonés. Collectanea Botanica 16, 391-395.

Montserrat Martí, G., Navarro del Águila, T., Gómez García, D, Maestro Martínez, M., Santamaría Pérez, B., Jiménez Jaén, J.J., Palacio Blasco, S. 2017. Estudio de la matorralización de pastos en el Parque Nacional de Ordesa-Monte Perdido (PNOMP). En: P. Amengual (Ed.), Proyectos de investigación en parques nacionales: 2012-2015. Red de Parques Nacionales Madrid, pp. 247-265.

Montserrat Martí, J. 1992. Evolución glaciar y postglaciar del clima y la vegetación en la vertiente sur del Pirineo: Estudio palinológico. Monografías del Instituto Pirenaico de Ecología 6, $147 \mathrm{pp}$.

Montserrat Recoder, P. 1964. Ecología del pasto. Ecología de los agrobiosistemas pastorales. Publicaciones del Centro pirenaico de Biología experimental 1, 1-68.

Morales-Molino, C., García-Antón, M. 2014. Vegetation and fire history since the last glacial maximumin an inland area of the western Mediterranean Basin (Northern Iberian Plateau, NW Spain). Quaternary Research 81, 63-77. https://doi.org/10.1016/j.yqres.2013.10.010.

Morales-Molino,C.,García-Antón,M.,Postigo-Mijarra,J.M.,Morla,C.2013.Holocene vegetation, fire and climate interactions on the westwernmost fringe of the Mediterranean Basin. Quaternary Science Reviews 59, 5-17. https://doi.org/10.1016/j.quascirev.2012.10.027.

Navarro, L.M., Pereira, H.M. 2012. Rewilding abandoned landscapes in Europe. Ecosystems 15, 900-912. https://doi.org/10.1007/s10021-012-9558-7.

Niven, L. 2007. From carcass to cave: Large mammal exploitation during the Aurignacian at Vogelherd, Germany. Journal of Human Evolution 53, 362-382. https://doi.org/10.1016/j. jhevol.2007.05.006.

Nogués-Bravo, D., Ohlemüller, R., Batra, P., Araújo, M.B. 2010. Climate predictors of late Quaternary extinctions. Evolution 64-68, 2442-2449. https://doi.org/10.1111/j.15585646.2010.01009.x.

Oliva, J., Colinas, C. 2007. Decline of silver fir (Abies alba Mill.) stands in the Spanish Pyrenees: Role of management, historic dynamics and pathogens. Forest Ecology and Management 252, 84-97. https://doi.org/10.1016/j.foreco.2007.06.017. 
Oliva,J.,Colinas, C. 2010. Epidemiology of Heterobasidion abietinum and Viscum album on silver fir (Abies alba).stands of the Pyrenees. Forest Pathology 40, 19-32. https://doi.org/10.1111/j.1439-0329.00603.x.

ORCA. Organització per a la cartografía de les plantes als països catalans. www.orca.cat

Owen-Smith, N. 1987. Pleistocene extinctions: the pivotal role of megaherbivores. Paleobiology 13, 351-362. https://doi.org/10.1017/S0094837300008927.

Owen-Smith, R.N. 1988. Megaherbivores. The influence of very large body size on ecology. Cambridge University Press.New York,369pp.https://doi.org/10.1017/CBO9780511565441.

Parducci, L., Jorgensen, T., Tollefsrud, M.M., Everland, E., Alm, T., Fontana, S.L., Bennett, K.D., Haile, J., Matetovici, I., Suyama, Y., Edwards, M.E., Andersen, K., Rasmussen, M., Boessenkool, S., Coissac, E., Brochmann, C., Taberlet, P., Houmark-Nielsen, M., Krog Larsen, N., Orlando, L., Gilbert, T.P., Kjaer, K.H., Greve Alsos, I., Willerslev, E. 2012. Glacial survival of boreal trees in northern Scandinavia. Science 335, 1083-1086. https://doi. org/10.1126/science.1216043.

Pellegrini, M., Donahue, R.E., Chenery, C., Evans, J., Lee-Thorp, J., Montgomery, J., Mussi, M. 2008. Faunal migration in late-glacial Italy; implications for human resource exploitation. Rapid Communications in Mass Spectrometry 22, 1714-1726. https://doi.org/10.1002.rcm.3521.

Pérez Obiol, R., Jalut, G., Julià, R., Pèlachs, A., Iriarte, M.J., Otto, T., Hernández-Beloqui, B. 2011. Mid-Holocene vegetation and climatic history of the Iberian Peninsula. The Holocene 21, 75-93. https://doi.org/10.1177/0959683610384161.

Pérez Romero, E. 2007. Factores zootécnicos en la crisis de la trashumancia castellana. Hispania 67, 1041-1068. https://doi.org/10.3989/hispania.2007.v67.i227.71.

Pérez Romero, E. 2016. El negocio de la trashumancia en el siglo XVIII: la cabaña de Rojas (17461808). Historia agraria 69, 43-72.

Pérez-Díaz, S., López-Sáez, J.A., Galop, D. 2015. Vegetation dynamics and human activity in the Western Pyrenean Region during the Holocene. Quaternary International 364, 65-77. https://doi.org/10.1016/j.quaint.2014.10.019.

Pokorny, P., Chytry, M., Juricková, L., Sádlo, J., Novák, J., Lozek, V. 2015. Mid-Holocene bottleneck for central European dry grasslands: Did steppe survive the forest optimum in northern Bohemia, Czech Republic? The Holocene 25, 716-726. https://doi. org/10.1177/0959683614566218.

Ponel, P. 1995. Rissian, Eemian and Würmian Coleoptera assemblages from La Grande Pile (Vosges, France). Palaeography, Palaeoclimatology, Palaeoecology 114, 1-41. https://doi. org/10.1016/0031-0182(95)00083-X.

Pott, R. 1995. The origin of grassland plant species and grassland communities in Central Europe. Fitosociologia 29, 7-32.

Power, M.J., Marlon, J., Ortiz, N., Bartlein, P.J., Harrison, S.P., Mayle, F.E., Ballouche, A., Bradshaw, R.H.W., Carcaillet, C., Cordova, C., Mooney, S., Prentice, I.C., Thonicke, K., Tinner W. and 69 more 2008. Changes in fire regimes since the Last Glacial Maximum: an assessment based on a global synthesis and analysis of charcoal data. Climate Dynamics 30, 887-907. https://doi.org/10.1007/s00382-007-0334-x

Pushkina, D., Raia, P. 2008. Human influence on distribution and extinctions of the late Pleistocene Eurasian megafauna. Journal of human evolution 54, 769-782. https://doi.org/10.1016/j. jhevol.2007.09.024.

Ramil-Rego, P., Rodríguez Guitián, M., Muñoz-Sobrino, C. 1998. Sclerophyllous vegetation dynamics in the north of the Iberian peninsula during the last 16,000 years. Global Ecology and Biogeography Letters 7, 335-351. https://doi.org/102307/2997682.

Ricánková, V.P., Horsák, M., Hais, M., Robovsky, J., Chytry, M. 2018. Environmental correlates of the Late Quaternary regional extinctions of large and small Paleartic mammals. Ecography 41, 516-527. https://doi.org/10.1011/ecog.02851. 
Richards, M.P. 2002. A brief review of the archaeological evidence for Palaeolithic and Neolithic subsistence. European Journal of Clinical Nutrition 56. https://doi.org/10.1038/ sj.ejen1601646. https://doi.org/10.1038/sj.ejcn.1601646.

Richards, M.P. 2009. Stable isotope evidence for Europaean Upper Paleolithic human diets. En: J.J. Hublin, M.P. Richards (Eds.), The evolution of hominin diets: integrating approaches to the study of Palaeolithic subsistence. Springer, pp. 251-257. https://doi.org/10.1007/978-14020-9699-0_20.

Rillardon, M., Brugal, J.P. 2014. What about the broad spectrum revolution? Subsistence strategy of hunter-gatherers in southeast France between 20 and 8 Ka BP. Quaternary International 337, 129-153. https://doi.org/10.1016/j.quaint.2014.01.020.

Rius, D., Vannière, B., Galop, D., Richard, H. 2011. Holocene fire regime changes from multiplesite sedimentary charcoal analyses in the Lourdes basin (Pyrenees, France). Quaternary Science Reviews 30, 1696-1709. https://doi.org/10.1016/j.quascirev.2011.03.014.

Roberts, N., Fyfe, R.M., Woodbridge, J., Gaillard, M.J., Davis, B.A.S, Kaplan, J.O., Marquer, L., Mazier, F., Nielsen, A.B., Suygita, S., Trondman, A.K., Leydet, M. 2018. Europe's lost forests: a pollen-based synthesis for the last 11,000 years. Scientific Reports 8, 716. https:// doi.org/10.1038/s41598-017-18646-7.

Robin, V., Nadeau, M.J., Grootes, P.M., Bork, H.R., Nelle, O. 2016. Too early too northerly: evidence of temperate trees in northern Central Europe during the Younger Dryas. New Phytologist 212, 259-268. https://doi.org/10.1111/nph.13844.

Ruiz de la Torre, J. 1979. Árboles y arbustos de la España peninsular. Escuela Técnica Superior de Ingenieros de Montes, Madrid, 512 pp.

Ruiz, M., Ruiz, J.P. 1986. Ecological history of transhumance in Spain. Biological Conservation 37, 73-86. https://doi.org/10.1016/0006-3207(86)90035-2.

Rule, S., Brook, B.W., Haberle, S.G., Turney, C.S.M., Kershaw, A.P, Johnson, C.N. 2012. The aftermath of megafaunal extinction: Ecosystem transformation in Pleistocene Australia. Science 335(6075), 1483-1486. https://doi.org/10.1126/science.1214261.

Sandom, C.J., Faurby, S., Sandel, B., Svenningn, J.C. 2014a. Global late Quaternary megafauna extinctions linked to humans, not climate change. Proceedings of the Royal Society BBiological Sciences 281, 1-9. https://doi.org/10.1098/rspb.2013.3254.

Sandom, J.S., Ejrnaes, R., Hansen, M.D.D., Svenning, J.C. 2014 b. High herbivore density associated with vegetation diversity in interglacial ecosystem. PNAS 111, 4162-4167. https:// doi.org/10.1073/pnas.1311014111.

Sangüesa Barreda, G., Camarero, J.J., Oliva, J., Montes, F. 2015. Past logging, drought and pathogens interact and contribute to forest dieback. Agricultural and Forest Meteorolgy 208, 85-94. https://doi.org/10.1016/j.agrformet.2015.04011.

Schnitzler, A. 2014. Towards a new European wilderness: Embracing unmanaged forest growth and the decolonisation of nature. Landscape Urban Planning 126:74-80. https://doi. org/10.1016/j.landurbanplan.2014.02.011.

Scholes, R.J., Archer, S.R. 1997. Tree-grass interactions in savannas. Annual Reviews of Ecology and Systematics 28, 517-544. https://doi.org/10.1146/annurev.ecolsys.28.1.517.

Schulting, R. 2011. Mesolithic-Neolithic transitions: An isotopic tour through Europe. En: R. Pinhasi, J. Stock (Eds.), Human Bioarchaeology of the transition to Agriculture. John Wiley \& Sons, Chichester, pp. 17-41. https://doi.org/10.1002/9780470670170.ch2.

Shea, J.J. 2006. The origins of lithic projectile point technology: evidence from Africa, the Levant, and Europe. Journal of Archaeological Science 33, 823-846. https://doi.org/10.1016/j. jas.2005.10.015.

Smith, F.A., Doughty, C.E., Malhi, Y., Svenning, J.C., Terborgh, J. 2016. Megafauna in the Earth system. Ecography 39, 99-108. https://doi.org/10.1111.ecog.02156. 
Smith, F.A., Elliott, S.M., Lyons, S.K. 2010. Methane emissions from extinct megafauna. Nature Geoscience 3, 374-375. https://doi.org/10.1038/hgeo877.

Soepboer, W., Lotter, A.F. 2009. Estimating past vegetation openness using pollen-vegetation relationships: a modelling approach. Review of Paleobotany and Palynology 153, 102-107. https://doi.org/10.1016/j.revpalbo.2008.07.004.

Sommer, R.S., Benecke, N., Lougas, L., Nelle, O., Schmölcke, U. 2011. Holocene survival of the wild horse in Europe: a matter of open landscape? Journal of Quaternary Science 26, 805812. https://doi.org/10.1002/jqs.1509.

Staver, A.C., Bond, W.J. 2014. Is there a "browse trap"? Dynamics of herbivore impacts on trees and grasses in an African savanna. Journal of Ecology 102, 595-602. https://doi. org/10.1111/1365-2745.12230.

Stoate, C., Báldi, A., Beja, P., Boatman, N.D., Herzon, I., Van Doom, A., de Snoo, G.R., Rakosy, L., Ramwell, C. 2009. Ecological impacts of early $21^{\text {st }}$ century agricultural change in Europe - A reiew. Journal of Environmental Management 91, 22-46. https://doi.org/10.1016/j. envman.2009.07.005.

Stuart, A.J. 2005. The extinction of woolly mammoth (Mammuthus primigenius) and straighttusked elephant (Palaeoloxodon antiquus) in Europe. Quaternary International 126-128, 171-177. https://doi.org/10.1016/j.quaint.2004.04021.

Stuart, A.J. 2015. Late Quaternary megafaunal extinctions on the continents: a short review. Geological Journal 50, 338-363. https://doi.org/10.1002/gj.2633.

Sugita, S. 2007a. Theory of quantitative reconstruction of vegetation I: pollen from large sites REVEALS regional vegetation composition. The Holocene 17, 229-241. https://doi. org/10.1177/0959683607075837.

Sugita, S. 2007b. Theory of quantitative reconstruction of vegetation II: al you need is LOVE. The Holocene 17, 243-257. https://doi.org/10.1177/0959683607075838.

Surovell, T., Waguespack, N., Brantingham, P.J. 2005. Global archaeological evidence for proboscidean overkill. Proceedings of the National Academy of Sciences of the United States of America 102, 6231-6236. https://doi.org/10.1073/pnas.0501947102.

Surovell, T.A., Pelton, S.R., Anderson-Sprecher, R., Myers, A.D. 2016. Test of Martin's overkill hypothesis using radiocarbon dates on extinct megafauna. Proceedings of the National Academy of Sciences of the United States of America 113, 886-891. https://doi.org/10.1073/ pnas. 1504020112 .

Svenning, J.C. 2002. A review of natural vegetation openess in north-western Europe. Biological Conservation 104, 133-148. https://doi.org/10.1016/S0006-3207(01)00162-8.

Svenning, J.C, Normand, S., Kageyama, M. 2008. Glacial refugia of temperate trees in Europe: insights from species distribution modelling. Journal of Ecology 96, 1117-1127. https://doi. org/10.1111/j.1365-2745.2008.01422.x.

Tinner, W., Conedera, M., Ammann, B., Lotter, A.F. 2005. Fire ecology north and south of the Alps since the last ice age. The Holocene 15, 1214-1226. https://doi. org/10.1191/0959683605hl892rp.

Tzedakis, P.C., Emerson, B.C., Hewitt, G.M. 2013. Cryptic or mystic? Glacial tree refugia in northern Europe. Trends in Ecology and Evolution 28, 696-704. https://doi.org/10.1016/j. tree.2013.09.001.

Uribe-Echebarría, P., Zorrakin, I. 2004. Claves ilustradas de la flora del Moncayo. Gobierno de Aragón. Departamento de Medio Ambiente, Zaragoza, 335 pp.

Valdés, B., Talavera, S., Fernández-Galiano, E., Eds. 1987. Flora de Andalucía Occidental. Ketres Editora, Barcelona.

Valdeyron, N. 2008. The Mesolithic in France. En: G. Pailey, P. Spikins (Eds.), Mesolithic Europe. Cambridge University Press, pp. 182-202. 
Vannière, B., Colombaroli, D., Chapron, E., Leroux, A., Tinner, W., Magny, M. 2008. Climate versus human-driven fire regimes in Mediterranean landscapes: the Holocene record of Lago dell'Accesa (Tuscany, Italy). Quaternary Science Reviews 27, 1181-1196. https://doi. org/10.1016/j.quascirev.2008.02.011.

Vannière, B., Power, M.J., Roberts, N., Tinner, W., Carrión, J.S., Magny, M., Bartlein, P., Colombaroli, D., Daniau, A.L., Finsinger, W., Gil-Romera, G., Kaltenrieder, P., Pini, R., Sadori, L., Turner, R., Valsecchi, V., Vescovi, E. 2011. Circum-Mediterranean fire activity and climate changes during the mid-Holocene environmental transition (8500-2500 cal. BP). The Holocene 21, 53-73. https://doi.org/10.1177/0959683610384164.

Vera, F.W.M. 2000. Grazing ecology and forest history. CABI Publishing, Oxon. https://doi. org/10.1079/9780851994420.0000.

West, R.G., Peglar, S.M., Pettit, M.E., Preece, R.C. 1995. Late Pleitocene deposits at Block Fen, Cambridgeshire, England. Journal of Quaternary Science 10, 285-310. https://doi. org/10.1002/jqs.3390100307.

Whitehouse, N.J. 1997. Insect faunas associated with Pinus sylvestris L. from the mid-Holocene of the Humberhead Levels, Yorkshire. En: A.C. Ashworth, P.C. Buckland, J.P. Sadler (Eds.), Studies in quaternary entomology- an inordinate fondness for insects. Quaternary Proceedings. John Wiley, Chichester, pp. 293-303.

Whitehouse, N.J., Smith, D. 2010. How fragmented was the British Holocene wildwood? Perspectives on the "Vera" debate from the fossil beetle record. Quaternary Science Reviews 29, 539-553. https://doi.org/10.1016/j.quascirev.2009.10.1010.

Willis, K.J., van Andel, T.H. 2004. Trees or no trees? The environments of central and eastern Europe during the Last Glaciation. Quaternary Science Reviews 23, 2369-2387. https://doi. org/10.1016/j.quascirev.2004.06.002.

Wing, S.L., Tiffney, B.H. 1987. Interactions of angiosperms and herbivorous tetrapods through time. En: E.M. Friis, W.G. Chaloner, P.R. Crane (Eds.), The origins of angiosperms and their biological consequences. Cambridge University Press, Cambridge, 203-24 pp.

Zaatari, S.E., Hublin, J.J. 2014. Diet of Upper Paleolithic modern humans: evidence from microwear texture analysis. American Journal of Physical Anthropology 153, 570-581. https://doi.org/10.1002/ajpa.22457.

Zalidis, G., Stamatiadis, S., Takavakoglou, V., Eskridge, K., Misopolinos, N. 2002. Impacts of agricultural practices on soil and water quality in the Mediterranean region and proposed assessment methodology. Agriculture, Ecosystems and Environment 88, 137-146.

Zanon, M., Davis, B.A.S., Marquer, L., Brewer, S., Kaplan, J.O. 2018. European forest cover during the past 12,000 years: a palynological reconstruction based on modern analogs and remote sensing. Frontiers in Plant Science 9 (253), 1-25. https://doi.org/10.1016/S0167-8809(01)00249-3.

Zhu, D., Ciais, P., Chang, J., Krinner, G., Peng, S., Viovy, N., Peñuelas, J., Zimov, S. 2018. The large mean body size of mammalian herbivores explains the productvity paradox during the Last Glacial Maximum. Nature, ecology and evolution. https://doi.org/10.1038/s41559-018-0481-y.

Zimov, S.A., Chuprynin, V.I., Oreshko, A.P., Chapin III, F.S., Reynolds, J.F., Chapin, M.C. 1995. Steppe-tundra transition: an herbivore-driven biome shift at the end of the Pleistocene. The American Naturalist 146, 765-794. https://doi.org/10.1086/285824.

Zimov, S.A., Zimov, N.S., Tikhonov, A.N., Chapin F.S. 2012. Mammoth seppe: A highproductivity phenomenon. Quaternary Science Reviews 57, 26-45. https://doi.org/10.1016/j. quascirev.2012.10.005.

Zorita, E. 2001. La investigación zootécnica española. Las razones de un fracaso. Archivos de Zootecnia 50, 441-463.

Zuo,W., Smith,F.A.,Charnov, E.L. 2013. A life-history approach to the late Pleistocene megafaunal extinction. The American Naturalist 182, 524-531. https://doi.org/10.1086/671995. 